\title{
Erythrocyte-derived Extracellular Vesicles Aggravates Inflammation via Facilitating pro- inflammatory Phenotype of Macrophages
}

\section{Yuhan Gao ( $\sim$ gaoyuhan1228@163.com )}

Peking University People's Hospital

Haiqiang Jin

Peking University First Hospital

Hui Tan

The First Affiliated Hospital of Shenzhen University

Ruiqin Hou

Peking University People's Hospital

Wenqin Tian

Peking University People's Hospital

Jianhua Zhang

Peking University People's Hospital

Ying Yang

Peking University People's Hospital

\section{Ranran Qin}

Peking University People's Hospital

\section{Yongan Sun}

Peking University First Hospital

\section{Research Article}

Keywords: morbidity , mortality , red blood cell (RBC), extracellular vesicles (EVs), transfusion-related immunomodulation (TRIM), caecal ligation and puncture (CLP)

Posted Date: January 25th, 2021

DOl: https://doi.org/10.21203/rs.3.rs-149389/v1

License: (c) (1) This work is licensed under a Creative Commons Attribution 4.0 International License.

Read Full License 
Original Article

Erythrocyte-derived extracellular vesicles aggravates inflammation via facilitating pro-inflammatory phenotype of macrophages

Yuhan Gao ${ }^{\text {a\# }}$, Haiqiang Jin ${ }^{\mathrm{b}}$, Hui Tan ${ }^{\mathrm{c}}$, Ruiqin $\mathrm{Hou}^{\mathrm{a}}$, Wenqin Tian ${ }^{\mathrm{a}}$, Jianhua Zhang ${ }^{\mathrm{a}}$, Ying Yang ${ }^{\mathrm{a}}$, Ranran Qin and Yongan Sun ${ }^{\mathrm{b} \#}$

${ }^{a}$ Department of Blood Transfusion, Peking University People's Hospital, Beijing, China

${ }^{b}$ Department of Neurology, Peking University First Hospital, Beijing, China

${ }^{\mathrm{c}}$ Department of Neurosurgery, The First Affiliated Hospital of Shenzhen University, Shenzhen, China.

\#To whom correspondence should be addressed at:

Yuhan Gao, Department of Blood Transfusion, Peking University People’s Hospital, Xizhimen

South Road 11, Xicheng District, Beijing, 100035, China, E-mail: gaoyuhan1228@163.com

And

Yongan Sun, Department of Neurology, Peking University First Hospital, No.8 Xishiku Street, Xicheng District, Beijing, 100034, China, E-mail: sya75@163.com 


\begin{abstract}
Transfusion of aged erythrocytes is associated with increased morbidity and mortality in the critically infections with incompletely understood mechanism. Previous studies suggested red blood cell (RBC)-derived extracellular vesicles (EVs) may be potential risk factors for the occurrence of transfusion-related immunomodulation (TRIM). The purpose of our study is to evaluate the effects of EVs under the inflammation condition and explore the underlying mechanisms. In vivo, the activity of EVs was evaluated in the caecal ligation and puncture (CLP)-induced sepsis. Our results showed EVs significantly aggravated the inflammatory response of sepsis in serum and lung tissue by promoting the production of pro-inflammatory factors, TNF- $\alpha$, IL6 , IL-1 $\beta$, and reduced the survival rate of septic mice in vivo. Importantly, adoptive transfer EVs pretreated bone marrow-derived macrophages (BMDM) obviously aggravated the systemic pro-inflammatory factors in mice after CLP surgery. In vitro, the pro-inflammatory properties of EVs were shown as elevating the levels of TNF- $\alpha$, IL-6, IL-1ßin LPS-stimulated BMDM. Moreover, EVs promoted the LPS-induced macrophages polarization into pro-inflammatory phenotype. The underlying mechanism was possibly that EVs could up-regulate NF-kB and MAPKs activity to favor macrophage cytokines production.
\end{abstract}




\section{Introduction}

In spite of advantages of packed red blood cell (RBC) transfusion, it also carries complications as well. Previous clinical and animal trials demonstrated that RBC transfusion could aggravate inflammations of the receptor, such as increasing the risk

of bacterial infection. ${ }^{1-5}$ Moreover, transfusion of aged erythrocytes is associated with increased morbidity and mortality in the critically infections with unknown cause. ${ }^{6-}$ ${ }^{8}$ Although the exact mechanism of transfusion-related immunomodulation (TRIM) is still not identified, previous studies suggested RBC-derived extracellular vesicles (EVs) may be potential risk factors for the occurrence of TRIM. ${ }^{9-13}$

RBC-derived EVs are sub-micron heterogeneous morphology vesicles, mediating an important role in the inflammatory response. ${ }^{14,15}$ Storage conditions and period, donor variability and manufacturing process can be related to the development of EVs. ${ }^{16,17}$ Straat showed that EVs from both fresh and stored RBC bags induced strong inflammatory response in the whole blood cells by elevated TNF- $\alpha$, IL-6 and IL-8 production in a dose-dependent manner. ${ }^{11}$ Danesh demonstrated that EVs not only induced production of predominantly pro-inflammatory cytokines and chemokines such as interleukin (IL)1, interferon gamma (IFN- $\gamma$ ), tumor necrosis factoralpha (TNF- $\alpha$ ) in peripheral blood mononuclear cells (PBMCs) but also improved the survival of PBMCs and augmented mitogen-driven T-cell responses at least in part through CD40/CD40L pathway. ${ }^{18}$ Microvesicles from aged erythrocytes could also amplify systemic inflammation, by enhancing release of pro-inflammatory cytokines IL-6, keratinocyte-derived chemoattractant (KC), and monocyte 
chemoattractant protein-1 (MCP-1) after injection in lipopolysaccharides (LPS) primed mice mainly through inducing thrombin-dependent complement activation. ${ }^{19}$ However, given the mixed cell population in the peripheral blood, it was still not clear the exact target(s) and mechanism(s) of RBC derived EVs.

Due to the widespread distribution and capacity of the cytokines production, macrophages play a pivotal role in infection, such as sepsis. ${ }^{20}$ Macrophages categorized as pro-inflammatory M1 macrophages and anti-inflammatory M2 macrophages. ${ }^{21-23} \mathrm{M} 1$ macrophage, induced by microbial factors like LPS or cytokines like IFN- $\gamma$, is characterized by increasedIL-1 $\beta$, IL- 6 and TNF- $\alpha$, and enhanced Th1 response. ${ }^{24}$ Alternatively, M2 macrophage, promoted by cytokines IL4 and IL-13, is characterized by up-regulation expression of arginase 1 (Arg1), Ym-1, and Found in Inflammatory Zone 1 (FIZZ1). ${ }^{25}$ When the tissue is infected or injured, macrophages are rapidly activated to phagocytize pathogens and exhibited a proinflammatory phenotype at the first few days. ${ }^{22,26}$ This pro-inflammatory cytokines and chemokine thereby mediate numerous of physiological and pathological response including recruitment and activation of lymphocytes and other phagocytes and activation of the adaptive immune system. ${ }^{26}$ However, excessive release of inflammatory mediators results in a hyper-inflammatory state, which leads to loss in control of inflammatory macrophage response. Therefore, inflammatory macrophage is particularly important for infection process. Among the inflammatory related pathways in macrophages, mitogen-activated protein kinase (MAPK) and nuclear transcription factor kappa $\mathrm{B}(\mathrm{NF}-\mathrm{\kappa} \mathrm{B})$ are significant contributors in immune 
responses. ${ }^{27}$ MAPK system included extracellular signal-regulated kinases (ERK1/2), stress-activated protein kinase/Jun amino-terminal kinase (SAPK/JNK) and the p38 MAPK. ${ }^{28,29}$ The activation of MAPKs and NF- $\mathrm{BB}$ can phosphorylate their substrates and transcription factors, which can activate the transcription of pro-inflammatory genes such as IL-1, IL-6 and TNF- $\alpha$.

Although many attempts have been made to examine the biological activities of EVs in stored $\mathrm{RBC}$ units, it is increasingly vital to investigate the details of this molecular targets and mechanisms to further strengthen the RBC transfusion indication and to avoid unexpected side-effects. Here, in the present study, we investigated the potential effect of RBC-derive EVs on macrophages as well as the underlying mechanism with mouse model of caecal ligation and puncture (CLP)-induced sepsis. 


\section{Results}

\section{Characterization of mice RBC-derived EVs}

EVs were isolated and characterized from the supernatant of stored leukocytedepleted, packed erythrocytes according to the method of Gao's et al study ${ }^{35}$.

\section{Effects of EVs on lung damage of CLP mice}

To evaluate the potential role of RBC-EVs in inflammatory conditions, the CLP mice model following by EVs transfusion as the second-hit was performed. Results from Fig. 1A showed that comparing to the sham-operated mice, EVs transfusion significantly increased the mortality of CLP mice. In spite of limited effect of EVs in the sham group, the expression of systemic pro-inflammatory cytokines, TNF- $\alpha$, IL-6, IL-1 $\beta$ was significantly up-regulated in the CLP mice after2 hours of EVs transfusion (Fig. 1B). The similar phenomenon was also observed in lung tissue, the major site of damage during the pathogenesis of sepsis (Fig. 1C). Infiltrated cells and macrophages in bronchoalveolar lavage fluid (BALF) was significantly increased after treatment with EVs as well (Fig. 1D, E). The results of flow cytometry showed that EVs increased proportion of CD11c macrophages from BALF (Fig. 1F). Histological analysis of the lung tissue also confirmed exacerbated pathological abnormality such as the damage of epithelial architecture, alveolar fluid exudation, and massive inflammatory cells infiltration in EVs treated CLP mice compared to PBS-treated CLP mice (Fig. 1G). On the other hand, there was normal histological architecture of 
lung in EVs treated sham-operated mice (Fig. 1G). In line with pathological analysis, the wet/dry ratio of lung tissue showed a similar trend (Fig. 1H).

\section{Effects of EVs on macrophage of CLP mice}

To further confirm the target cells of EVs in vivo, macrophage-depleted mice with clodronate liposomes were adoptively transferred pretreated bone marrow derived macrophage (BMDM) and then performed the CLP. The macrophages were almost depleted in the clodronate liposome treatment mice at 24 hours (Fig. 2A). Then, the pretreated BMDMs were adoptive transferred to these depleted mice. As shown in Fig. 2B, the LPS treated BMDMs obviously decreased the survival rate of CLP mice. Furthermore, EVs pretreatment exacerbated the disease and deteriorated the survival. In addition, the serum pro-inflammatory cytokines (TNF- $\alpha$ and IL-1 $\beta$ ) induced by the pro-inflammatory phenotypic BMDM, were dramatically elevated in mice receiving

EVs-exposed BMDMs (Figure 2C). Large numbers of macrophages migrated into the abdominal cavity, and these inflammatory response could be aggravated in mice receiving EVs-exposed pro-inflammatory phenotypic BMDMs (Fig. 2D). Taken together, these data suggested that the pro-inflammatory effect of EVs in vivo might target macrophages and facilitate its polarization towards pro-inflammatory phenotype.

\section{Effect of EVs on cell viability}

The potential cytotoxicity of EVs on BMDM was investigated by the MTT (3-(4,5Dimethylthiazol-2-yl)-2,5-Diphenyltetrazolium Bromide) assay and CFSE (carboxy 
fluorescein diacetate succinimide ester) staining. At the concentrations from 0.1$2.5 \mu \mathrm{g} / \mathrm{ml}$, EVs had negligible effect on the cells viability and proliferation (Fig. 3). Herein, in order to exclude the potential influence of cytotoxin, the concentration of EVs was limited to $2.5 \mu \mathrm{g} / \mathrm{ml}$ in the following study.

\section{Effects of EVs on LPS-induced TNF- $\alpha$, IL-6, IL-1ß and IL-10 production}

Macrophages are immune cells which release various cytokines in response to LPS stimulation. To investigate the potential effect of EVs on the macrophage cytokines production, TNF- $\alpha$, IL- 6 , IL- $1 \beta$ and IL-10 secretion were detected in the cell culture media by enzyme-linked immunosorbent assay (ELISA) while their messenger ribonucleic acid (mRNA) levels were quantified in cell lysates by quantitative reverse transcription-polymerase chain reaction(RT-PCR), respectively. As shown in Fig.

4A-C, BMDM treated with LPS displayed a pro-inflammatory phenotype characterized by dramatic increased of TNF- $\alpha$, IL-6, IL-1ßat protein and mRNA level. A slight but statistically insignificant increase was observed when BMDM cultured with EVs alone. Moreover, synergistic effect was observed between LPS and EVs on the TNF- $\alpha$, IL-6, IL-1 $\beta$ production. Notably, the anti-inflammatory mediators IL-10 remained unaltered on both protein and mRNA level when the BMDM cultured with EVs (Fig. 4D). These results suggested that EVs effectively promoted the production of pro-inflammatory cytokines in both resting and LPS- stimulated BMDM cells.

\section{Effects of EVs on macrophage polarization}


As macrophage polarization plays a key role in its function, mouse BMDM were collected and cultured to evaluate the effect of EVs on macrophage polarization phenotype. In classical activation of macrophages, EVs treatment increased the mRNA levels of inos, Tnf- $\alpha$ and Il-6, the M1 phenotype markers, in the stimulation of LPS and IFN- $\gamma$ (Fig. 5A-C). However, the mRNA levels of Arg-1, Ym-1 and Fizzl, which are characteristic M2 phenotype markers, were not affected by EVs under the stimulation with IL-4 (Fig. 5D-F). Next, the phagocytic capacity of macrophages was evaluate by fluorescein-labeled E. coli, and result showed higher fluorescence intensity was observed in the group adding EVs compared with the group of $E$. coli alone, suggesting the promoting function of EVs in LPS induced phagocytic function (Fig. 5G).

\section{Effects of EVs on LPS-induced activation of NF-אB and MAPK signaling}

Next, we sought to investigate how EVs signals to BMDM cells and how the EVsmediated signal is integrated with LPS stimulation. It is well known that NF- $\kappa \mathrm{B}$ is a transcription factor typically induced by LPS and the main regulator of proinflammatory cytokines in macrophages. ${ }^{30}$ As shown in Fig. 6A-C, EVs alone could moderately trigger the I $\kappa \mathrm{B} \alpha$ degradation and NF- $\mathrm{B}$ p 65 phosphorylation.

Furthermore, the degradation of $\mathrm{I} \kappa \mathrm{B} \alpha$ and phosphorylation of NF- $\kappa \mathrm{B}$ p 65 were further enhanced in combination of EVs and LPS. These findings indicated that the EVs treatment could promote the activation of NF- $\mathrm{B}$, with a significant synergistic effect between EVs and LPS. 
Except for the NF- $\mathrm{BB}$ signaling, the phosphorylation and activation status of kinases in the MAPK system have crucial impact on cytokines production of macrophage. ${ }^{31}$ So, the phosphorylation of kinases in the MAPK system was further examined. As shown in Fig. 6A, D-F, either EVs or LPS induced significant phosphorylation of p38, ERK and JNK, and in combination they induced even higher levels of phosphorylated p38, ERK and JNK. Therefore, EVs treatment could improve the phosphorylation and activation of MAPK system.

To evaluate the involvement of NF-אB and MAPK in LPS challenged macrophages, their inhibitors were used. BMDM were pretreated with Pyrrolidinedithiocarbamate (PDTC, NF- $\kappa$ B inhibitors), SB203580 (p38 inhibitors), U0126 (ERK inhibitors), or SP600125 (JNK inhibitors) for 30 min, followed by challenged with LPS or EVs for 24 hours. The production of TNF- $\alpha$ in the cell culture media was measured by ELISA. As shown in Fig. 6G, the inhibitors can inhibit the secretion of TNF- $\alpha$ caused by EVs to varying degrees. A similar tendency is also observed in the combination of EVs and LPS. 


\section{Discussion}

The impetus for this work was the clinical observation that transfusion of aged erythrocytes is associated with increased morbidity and mortality in the critically infections with incompletely understood mechanism. In our study, the proinflammatory activity of RBC-derived EVs on infection disease was identified and the underlying mechanisms were further elucidated for the first time. RBC-derived EVs, mainly taking advantages of the NF- $\mathrm{BB}$ and MAPK signaling pathways, prompted the pro-inflammatory phenotype of macrophages thus aggravating the inflammatory conditions in CLP-induced sepsis.

Firstly, the pro-inflammatory activity of EVs was evaluated in vivo. It is vital to rebalance the inflammatory response and prevent the organ dysfunction and the sequential organ failure in infection illness. RBC-derived EVs obviously reduced survival rate of mice with CLP-induced sepsis. This was accompanied by aggravated systemic pro-inflammatory factors in serum of sepsis model mice (Fig.1 A, B). EVs intensified the damage of lung tissue and increased the infiltration of proinflammatory macrophages (Fig.1). The process of inflammation was closely related to the phenotype of macrophages. ${ }^{32,33}$ Therefore, further investigation indicated that high expressed CD11c, a marker of pro-inflammatory macrophages, was significantly up-regulated by EVs treatment in lung tissue of CLP-induced sepsis mice (Fig.1F). These results hinted us that the pro-inflammatory activity of EVs for sepsis may be related to the regulation of macrophage polarization into pro-inflammatory phenotype. Furthermore, the targeting of macrophage by EVs in vivo was further confirmed in 
our experiments using adoptive transfer of EVs pretreated-BMDMs to macrophagedepleted mice (Figure 2). This pro-inflammatory properties of EVs, however, was barely observed in normal mice suggesting that EVs function may be mild and body could compensate for itin the healthy mice. However, EVs' action could be amplified by LPS infection.

Next, the activity of RBC-derived EVs on macrophage was evaluated in vitro. As shown in Fig. 3, the BMDM retained almost the same viability exposed to EVs at concentrations less than $2.5 \mathrm{mM}$ in 24 hour so that the effect of EVs on cell viability is excluded in subsequent studies. RBC-derived EVs increased both the mRNA and protein levels of pro-inflammatory factors (TNF- $\alpha$, IL-6 and IL-1 $\beta$ ) on its own in a moderate degree, and this promotion effect was more prominent under LPS stimulation (Fig. 4). Furthermore, the result of RT-PCR suggested EVs could promote LPS and IFN- $\gamma$ polarized into pro-inflammatory phenotype with increased mRNA expression of inos, Tnf- $\alpha$ and Il-6, but not IL-4 polarized into antiinflammatory phenotype with unchanged mRNA expression of Ym-1, Arg-1 and Fizzl in macrophages (Fig. 5). Other functional characteristics of these macrophages, such as phagocytosis, were also inhibited, further confirming the pro-inflammatory activity of EVs (Fig.5). This selectivity for the pro-inflammatory, over the antiinflammatory phenotype might aggravate the infection as the secondary-hit for the sepsis patients which received blood transfusion therapy.

In the previous studies, Zecher and co-workers ${ }^{19}$ found RBC-derived microvesicles could amplify both pulmonary leukocyte sequestration and induce higher serum 
levels of pro-inflammatory cytokines, such as IL-6, keratinocyte-derived chemoattractant (KC), and monocyte chemoattractant protein-1 (MCP-1) which was consistent with our in vivo data. However, in his study, administration of microvesicles in LPS-primed mice did not lead to invasive lung injury. In our experiments, RBC-derived EVs not only intensified the damage of lung tissue, but also obviously reduced survival rate of mice with CLP-induced sepsis. The possible reason is that 1) the differences in RBC and erythrocyte-derived EVs purification and storage conditions; 2) the differences in infection models. In our study, we chose the CLP-induced sepsis model which is much more similar to clinical endotoxin infection. In terms of mechanistic studies, they focused on the role of microvesicles in the complement activation. However, our point was the effect of EVs on macrophage, which played an important role in sepsis.

To confirm the underlying mechanism(s) and the molecular target(s) of EVs, we measured the phosphorylation levels of NF- $\mathrm{BB}$ and MAPKs pathways. Results in Figure 6 suggested EVs slightly enhanced the activation of NF- $\kappa B$ through promotion of $\mathrm{I} \kappa \mathrm{B} \alpha$ degradation and phosphorylation of NF- $\mathrm{BB}$ p65 subunit. And LPS-mediated NF- $\mathrm{BB}$ activity increased significantly by EVs synergistically. Furthermore, the elevated TNF- $\alpha$ secretion by EVs treatment can be inhibited through NF- $\kappa$ B inhibitor PDTC. Based on these results, our data indicated the enhanced pro-inflammatory cytokines by RBC-derive EVs was mediated, at least in part, via up-regulation of NF$\kappa \mathrm{B}$ activation and transactivation activity. Apart from NF- $\kappa \mathrm{B}$ pathway, MAPKs, highly conserved kinases family including ERK, p38 and JNK, are reported to be 
involved in pro-inflammation cytokines secretion in macrophages. ${ }^{31,34}$ Based on our study, RBC-derived EVs evidently increased phosphorylation of p38, ERK and JNK on its own. In addition, LPS induced ERK, p38 and JNK phosphorylation was further enhanced by EVs obviously (Fig. 6). Similarly, the EVs' function could also be inhibited by the respective inhibitors, SB203580, U0126, and SP006125. These results suggest that the increased activation of p38, ERK, and JNK may be involved in RBC-derive EVs induced cytokines production.

There were also shortcomings in our study. Firstly, the exact substance(s) of EVs that causes the macrophage polarization is not known. The RBC-derived EVs was collected and purified from packed leuko-reduced mouse RBC units. Erythrocyte separation and purification is conducted immediately after the whole blood collection finished. During this process, most white blood cells, platelets and plasma have been removed through filtration,centrifugation and repeat washing. There is no contamination with leukocyte-derived EVs and platelet-derived EVs in our study. ${ }^{35}$ There are several studies about the ingredient of red blood cell (RBC)-derived EVs. ${ }^{36,}$ ${ }^{37}$ It is reported that the main element of this EVs are actin, band 3, hemoglobin and lipid raft proteins (such as stomatin, flotillin-2). Scarce studies reported that EVs contained theToll-like receptors(TLRs) or other pathogen-associated molecular pattern (PAMP) in itself. So, the accurate substance(s) caused macrophage polarization still needs further experimental in the future. Besides, the exact molecular mechanism and the target of EVs arestill unclear and need investigation in further study. In our research, both NF-kB and MAPK pathway were affected, which 
inferred us that EVs' target might be the upstream of those two pathways, such as TLR4 and MyD88. Thirdly, other leukocytes are still vital in the process of sepsis, such as neutrophils and monocytes. Whether the RBC-derived EVs have effects on these cells needs further verification. Taking into consideration of the macrophagedepletion mice experiment and in vitro assay, EVs do promote macrophage polarization to pro-inflammatory phenotype. Finally, these studies have all been conducted with mouse RBC-derived EVs and an assessment of their effects on mouse macrophages. Whether it is also true for the EVs derived from human RBCs have the same effects needs further study.

In sum, the current study provided the evidence of pro-inflammatory effects of RBCderived EVs by interfering macrophage phenotypic polarization in sepsis which deepened the understanding of biological and molecular functions of RBC-derived EVs and gained further insight into the mechanismof TRIM. Furthermore, our data identified a new therapeutic direction for inflammatory patients requiring blood transfusion. 


\section{Materials and methods}

\section{Purification and quantified of EVs}

Packed leukoreduced mouse RBC units (Bestgene biotech company,zhengzhou, Henan) were stored at $4{ }^{\circ} \mathrm{C}$ for 21 days. EVs were isolated andpurified as described before. $^{35}$

\section{Mice and ethical statement}

All the study was carried out in compliance with the ARRIVE guidelines. The C57BL/6J mice between 6-8 weeks of age were purchased from the Beijing Vital River Laboratory Animal Technology Co. Ltd and were housed in a specific pathogen-free barrier facility with restricted access. Animal care and experimentation were performed in accordance with the standard guidelines for the care and use of experiment animals in Peking University People's Hospital. Besides, all the experimental protocols were approved by ethics committee of Peking University People's Hospital.

\section{Caecum ligation and puncture (CLP)-induced sepsis}

For the CLP induced sepsis mouse model, CLP surgery was performed as described previously. ${ }^{38}$ Briefly, mice were anaesthetized with $2 \%$ sodium pentobarbital (150 $\mu$ Lper 20 g) i.p.. The caecum was exposed carefully and ligated tightly with a 3.0 silk suture at the middle and punctured through-and-through with a 25 -gauge needle at 
two sites. A small amount of faecal material was pushed gently to the peritoneal cavity. After surgery, prewarmed $\left(37^{\circ} \mathrm{C}\right) 0.9 \%$ saline $(2 \mathrm{~mL})$ was injected to resuscitate mice immediately. For the sham group, mice were operated similarly without ligation and puncture. After 2 hours, the mice were injected with sterile PBS or EVs $(10 \mu \mathrm{g} / 0.1 \mathrm{ml} \mathrm{PBS})$ via tail vein. For the survival experiments, the mice were closely observed every $6 \mathrm{~h}$ for 2 days. For other experiments, the mice were sacrificed $6 \mathrm{~h}$ after the CLP surgery.

\section{Generation of bone marrow-derived macrophages (BMDM)}

The tibia and femur of hind legs were detached from 6- to 8-week-old C57BL/6 J mice. The bone marrow (BM) was flushed with $10 \%$ fetal bovine serum (FBS) using $5 \mathrm{ml}$ syringe, and the cells were resuspended by red blood cell lysis buffer at $4{ }^{\circ} \mathrm{C}$ for $5 \mathrm{~min}$. The BM cells were then cultured in $10 \mathrm{~cm}$ dish at a starting density of $5 \times$ $10^{5} \mathrm{cells} / \mathrm{mL}$ at $37^{\circ} \mathrm{C}$ in a humidified atmosphere of $5 \% \mathrm{CO}_{2}$ and differentiated into macrophages in Dulbecco's modified Eagle medium (DMEM) supplemented with $10 \%$ FBS, $1 \%$ penicillin/ streptomycin (Life Technologies, Grand Island, NY), and $50 \mathrm{ng} / \mathrm{mL}$ recombinant murine macrophage colony-stimulating factor (M-CSF; R\&D Systems, Minneapolis, MN) for 7 days. The macrophage phenotype was confirmed by flow cytometry with antibodies specific for F4/80 (BM8; eBioscience, San Diego, $\mathrm{CA})$ and $\mathrm{CD} 11 \mathrm{~b}^{+}$(M1/70; eBioscience).

\section{Cell culture and treatment}


Cultures of BMDM were stimulated with $0.1 \mu \mathrm{g} / \mathrm{mL}$ LPS(Sigma-Aldrich, St. Louis, $\mathrm{MO})$ or varying concentrations of EVs $(0.1-10 \mu \mathrm{g} / \mathrm{mL})$ for indicated time.

For the macrophage phenotypic polarization assay, BMDMs were treated with EVs $(2.5 \mu \mathrm{g} / \mathrm{mL})$ or the same volume of PBS in the presence of $0.1 \mu \mathrm{g} / \mathrm{mL}$ LPS (SigmaAldrich) and 20 ng/mL IFN- $\gamma$ (PeproTech, Rocky Hill, NJ) or 20 ng/mL IL-4 (PeproTech) for 24 hours induce pro-inflammatory and anti-inflammatory phenotype.

For the inhibition assay, the BMDM was cultured with NF- $\kappa$ B inhibitor ammonium pyrrolidinedithiocarbamate (PDTC, $25 \mu \mathrm{M}$, Sigma-Aldrich), p38 inhibitor SB203580

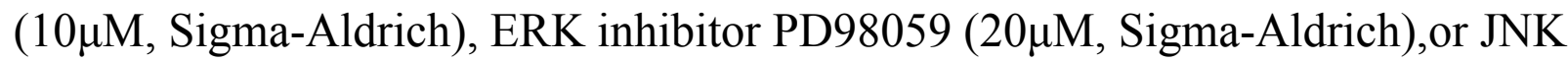
inhibitor SP600125 $(10 \mu \mathrm{M}$, Sigma-Aldrich) followed by LPS $(0.1 \mu \mathrm{g} / \mathrm{mL})$ or EVs $(2.5 \mu \mathrm{g} / \mathrm{mL})$ for 24 hours at $37^{\circ} \mathrm{C}$ with $5 \% \mathrm{CO}_{2}$ in humidified incubator.

\section{Cell viability assay}

The effect of EVs on cell viability was examined by MTT(3-(4,5-Dimethylthiazol-2yl)-2,5-Diphenyltetrazolium Bromide, Sigma-Aldrich) assay as manufacturer indicated. Briefly, BMDM $\left(5 \times 10^{5} \mathrm{cells} / \mathrm{ml}\right)$ were plated onto $96-$ well plates and exposed to PBS (as negative control) and varying concentrations of EVs (0.1$10 \mu \mathrm{g} / \mathrm{mL})$ for $24 \mathrm{~h} . \mathrm{MTT}(0.1 \mathrm{mg} / \mathrm{ml})$ was added to each well, and the cells were incubated for another $4 \mathrm{~h}$ at $37{ }^{\circ} \mathrm{C}$ with $5 \% \mathrm{CO}_{2}$ in humidified incubator. The medium was removed, and $100 \mu \mathrm{L}$ of DMSO (dimethyl sulfoxide) was added to each well to solubilize any deposited formazan. The optical density (OD) of each well was measured at $570 \mathrm{~nm}$ with a microplate reader (Molecular Devices, Sunnyvale, CA). 


\section{Proliferation assay}

For proliferation assay, $5 \times 10^{5} / \mathrm{ml} \mathrm{BMDM}$ was incubated at room temperature for 3 min in $1 \mathrm{ml}$ of PBS containing $5 \mu \mathrm{M}$ carboxy fluorescein diacetate succinimide ester (CFSE, Invitrogen, Carlsbad, CA). Cells were washed twice to remove free dye before being put into culture under various conditions as described above. After 3 days, cells were monitored for CFSE dilution using flow cytometry.

\section{Phagocytosis assay}

For phagocytosis assay, BMDM was exposed to FITC labeled E. coli. and $2.5 \mu \mathrm{g} / \mathrm{mL}$ EVs or PBS as negative control overnight. The phagocytic activity of macrophages was measured using flow cytometry.

\section{Quantitative reverse transcription-polymerase chain reaction (RT-PCR)}

Macrophages $\left(5 \times 10^{5} / \mathrm{ml}\right)$ were stimulated as indicated. Total RNA was isolated from the cultured cells using TRIzol reagent (Invitrogen) according to the manufacturer's protocol. Two micrograms of RNA were reverse transcribed to cDNA using oligo-dT primers and the Reverse Transcription System (Promega, Madison, WI). Quantitative RT-PCR reactions were performed in triplicate for each sample using iQ SYBR Green Supermix (Bio-Rad Laboratories, Hercules, CA). The quantification was based on $\triangle \mathrm{CT}$ and calibrated to the level in LPS-treated BMDM. $\beta$-actin was used as an internal control. Primers were used as follow: TNF- $\alpha$ (F:

CCGCTCGTTGCCAATAGTGATG; R: CATGCCGTTGGCCAGGAGGG), IL-6(F: ACAAAGCCAGAGTCCTTCAGAG; R: GGTCCTTAGCCACTCCTTCTG), IL- 
1ß(F: GCCACCTTTTGACAGTGATGAG; R: TGATACTGCCTGCCTGAAGC), IL-10(F: CCCTGGGTGAGAAGCTGAAG; R:

CACTGCCTTGCTCTTATTTTCACA), Inos (F: ACATCGACCCGTCCACAGTAT; R:CAGAGGGGTAGGCTTGTCTC), Arg-1 (F:

TGTCCCTAATGACAGCTCCTT;R:GCATCCACCCAAATGACACAT),Ym-1 (F:CAGGTCTGGCAATTCTTCTGAA;R:GTCTTGCTCATGTGTGTAAGTGA), Fizzl

(F:CCAATCCAGCTAACTATCCCTCC;R:ACCCAGTAGCAGTCATCCCA), $\beta$ actin(F: TGCTGTCCCTGTATGCCTCT; R: TTTGATGTCACGCACGATTT).

\section{Enzyme-linked immunosorbent assay (ELISA)}

BMDM was seeded in 12-well plates at $1 \times 10^{6}$ cells/well and cultured with LPS or $2.5 \mu \mathrm{g} / \mathrm{mL}$ EVs or both for 24 hours. The culture supernatants were collected to detect the TNF- $\alpha$, IL- 6 , IL-1 $\beta$ and IL-10 levels using the corresponding ELISA kit according to the manufacturer's instructions (R\&D Systems). Blood samples and bronchoalveolar lavage fluid (BALF)from CLP surgery mice were incubated at $37^{\circ} \mathrm{C}$ for $30 \mathrm{~min}$ and then centrifuged at $1500 \mathrm{~g}$ for $10 \mathrm{~min}$ at $4^{\circ} \mathrm{C}$. The serum of blood and supernatants of BALF cytokines, TNF- $\alpha$, IL- 6 andIL-1 $\beta$ were measured using ELISA kits. All samples were examined in triplicate.

\section{Cell counts in bronchoalveolar lavage fluid (BALF) and peritoneal cavity}

Immediately after intraperitoneal injection with an overdose of pentobarbital (50 $\mathrm{mg} / \mathrm{kg}$ ), $0.7 \mathrm{ml}$ of ice-cold PBS was instilled into the lung via the tracheal cannula 
and recovered by gentle manual aspiration. BALF collected from 3 repeats (total volume $2 \mathrm{ml}$ ) was pooled and centrifuged.

For the peritoneal macrophages, mice were killed and the peritoneal cavity was lavaged with $3 \mathrm{~mL}$ of PBS twice and centrifuged. The total number of inflammatory cells were counted using cell counting chamber (Yuanmu Biotechnologies, Shanghai,

China). The percentage of F4/ 80 macrophages were analyzed by flow cytometry.

\section{Lung Wet-to-Dry Weight Ratio}

The left lung was excised carefully, washed in PBS, dried using blotting paper gently, and then weighed. The tissue was then dried at $60^{\circ} \mathrm{C}$ for $72 \mathrm{~h}$ and reweighed. The change in the ratio of wet weight to dry weight was used as an indicator of lung edema formation.

\section{Lung tissue histopathology}

The lung tissue from CLP mice was fixed in 10\% neutral buffered formalin for 24 hours, and then embedded in paraffin, sectioned at $4 \mu \mathrm{m}$ thickness, followed by staining with hematoxylin and eosin (H\&E) to examine cell infiltration.

\section{Macrophage depletion and BMDM adoptive transfer assay}

Clodronate liposomes $(100 \mu \mathrm{L}$ per $20 \mathrm{~g}$ body weight; FormuMax Scientific Inc., Silicon Valley, CA) or PBS liposomes (FormuMax Scientific Inc.) were injected i.p. to induce systemic macrophage depletion in $\mathrm{C} 57 \mathrm{BL} / 6$ mice as described before. ${ }^{39}$ 
The proportion of $\mathrm{F} 4 / 80$ positive macrophage populations in the peritoneal cavity was analyzed by flow cytometry to detect the clearance effect 24 hours later.

BMDM was polarized to the pro-inflammatory phenotype with LPS $(0.1 \mu \mathrm{g} / \mathrm{mL})$ and IFN- $\gamma(20 \mathrm{ng} / \mathrm{mL})$ and incubated with $2.5 \mu \mathrm{g} / \mathrm{mL}$ EVs or the same volume of PBS for 6 hours(M1-BMDM). Normal BMDM (M0-BMDM) received the same volume of PBS. The pretreated cells were harvested and washed three times with cold PBS and then transferred to the macrophage-depleted mice $\left(1.5 \times 10^{6}\right.$ cells per $20 \mathrm{~g} ; 100 \mu \mathrm{L}$ per mouse, i.v.) $2 \mathrm{~h}$ before CLP surgery.

\section{Western blots}

BMDM cultured under various conditions were harvested and cell lysates were prepared in lysis buffer. The lysates were resolved on a $10 \%$ reducing SDSpolyacrylamide gels and electrophoretically transferred to a polyvinylidene difluoride membrane. After blocking with 3\% Bovine Serum Albumin (BSA; Sigma-Aldrich) for $1 \mathrm{~h}$, the membrane was incubated withanti-phospho-p65, anti-phospho-p38, antip38, anti-IкB $\alpha$, anti-phospho-p44/p42 MAPK (Erk1/2), anti-phospho-SAPK/JNK, anti-p44/p42 MAPK, anti-SAPK/JNK, or anti- $\beta$-actin (Cell Signaling Technology, Beverly, MA), overnight at $4{ }^{\circ} \mathrm{C}$ and then incubated with a Horseradish Peroxidase (HRP) coupled secondary antibody (Sigma Aldrich). The immunoreactive bands were detected by chemiluminescence with enhanced chemiluminescence (ECL) detection reagents (Life Technologies).

\section{Statistics}


Data are presented as mean \pm the standard error of the mean (SEM) unless otherwise specified. For statistical analysis, unpaired Student's t test and One-way ANOVA analysis was performed appropriatly using GraphPad Prism software (GraphPad Software, La Jolla, CA). The level of significance was set at a P value of 0.05 .

Acknowledgements: This work was supported by grant from the National Natural Science Foundation of China (No. 31700767, 82071306 and No.51773119), and Science Foundation of Guangdong Province (2019A1515011750). There are no conflicts of interest.

Author contributions: Yuhan Gao, Haiqiang Jin and Hui Tan performed experiments, analyzed and interpreted data and wrote the manuscript. Wenqin Tian, Jianhua Zhang, Ying Yang, Ruiqin Hou and Ranran Qin interpreted data. Yuhan Gao and Yongan Sun supervised the study and provided financial support. All authors revised the manuscript and approved its final version.

Competing Interests: The authors have no conflict of any interest relevant to this topic. The corresponding authors had full access to the data and had final responsibility for the decision to submit for publication.

Data availability statement: Any data of this study was available to be public if necessary. 


\section{Reference}

1. Vamvakas, E.C. \& Blajchman, M.A. Transfusion-related immunomodulation (TRIM): an update. Blood Rev21, 327-348 (2007).

2. Bilgin, Y.M. \& Brand, A. Transfusion-related immunomodulation: a second hit in an inflammatory cascade? Vox Sang95, 261-271 (2008).

3. Ferraris, V.A., Ballert, E.Q. \& Mahan, A. The relationship between intraoperative blood transfusion and postoperative systemic inflammatory response syndrome. Am J Surg205, 457-465 (2013).

4. Refaai, M.A. \& Blumberg, N. Transfusion immunomodulation from a clinical perspective: an update. Expert Rev Hematol6, 653-663 (2013).

5. Remy, K.E. et al. Mechanisms of red blood cell transfusion-related immunomodulation. Transfusion58, 804-815 (2018).

6. Ho, J., Sibbald, W.J. \& Chin-Yee, I.H. Effects of storage on efficacy of red cell transfusion: when is it not safe? Crit Care Med31, S687-697 (2003).

7. Hod, E.A. \& Spitalnik, S.L. Harmful effects of transfusion of older stored red blood cells: iron and inflammation. Transfusion51, 881-885 (2011).

8. Spitalnik, S.L. Stored red blood cell transfusions: iron, inflammation, immunity, and infection. Transfusion54, 2365-2371 (2014). 
9. Almizraq, R.J., Seghatchian, J. \& Acker, J.P. Extracellular vesicles in transfusion-related immunomodulation and the role of blood component manufacturing. Transfus Apher Sci55, 281-291 (2016).

10. Antonelou, M.H. \& Seghatchian, J. Update on extracellular vesicles inside red blood cell storage units: Adjust the sails closer to the new wind. Transfus Apher Sci55, 92-104 (2016).

11. Straat, M., Boing, A.N., Tuip-De Boer, A., Nieuwland, R. \& Juffermans, N.P. Extracellular Vesicles from Red Blood Cell Products Induce a Strong ProInflammatory Host Response, Dependent on Both Numbers and Storage Duration. Transfus Med Hemother43, 302-305 (2016).

12. Fischer, D. et al. Microparticles from stored red blood cells enhance procoagulant and proinflammatory activity. Transfusion57, 2701-2711 (2017).

13. Huang, H. et al. MicroRNA Profiling of Exosomes Derived from Red Blood Cell Units: Implications in Transfusion-Related Immunomodulation. Biomed Res Int2019, 2045915 (2019).

14. Nguyen, D.B. et al. Characterization of Microvesicles Released from Human Red Blood Cells. Cell Physiol Biochem38, 1085-1099 (2016).

15. Wannez, A. et al. Extracellular Vesicles in Red Blood Cell Concentrates: An Overview. Transfus Med Rev33, 125-130 (2019). 
16. Bicalho, B., Pereira, A.S. \& Acker, J.P. Buffy coat (top/bottom)- and wholeblood filtration (top/top)-produced red cell concentrates differ in size of extracellular vesicles. Vox Sang109, 214-220 (2015).

17. Almizraq, R.J., Holovati, J.L. \& Acker, J.P. Characteristics of Extracellular Vesicles in Red Blood Concentrates Change with Storage Time and Blood Manufacturing Method. Transfus Med Hemother45, 185-193 (2018).

18. Danesh, A. et al. Exosomes from red blood cell units bind to monocytes and induce proinflammatory cytokines, boosting T-cell responses in vitro. Blood123, 687696 (2014).

19. Zecher, D., Cumpelik, A. \& Schifferli, J.A. Erythrocyte-derived microvesicles amplify systemic inflammation by thrombin-dependent activation of complement. Arterioscler Thromb Vasc Biol34, 313-320 (2014).

20. Stearns-Kurosawa, D.J., Osuchowski, M.F., Valentine, C., Kurosawa, S. \& Remick, D.G. The pathogenesis of sepsis. Annu Rev Pathol6, 19-48 (2011).

21. Cassetta, L., Cassol, E. \& Poli, G. Macrophage polarization in health and disease. ScientificWorldJournal11, 2391-2402 (2011).

22. Mege, J.L., Mehraj, V. \& Capo, C. Macrophage polarization and bacterial infections. Curr Opin Infect Dis24, 230-234 (2011).

23. Yunna, C., Mengru, H., Lei, W. \& Weidong, C. Macrophage M1/M2 polarization. Eur J Pharmacol877, 173090 (2020). 
24. Mills, C.D., Kincaid, K., Alt, J.M., Heilman, M.J. \& Hill, A.M. M-1/M-2 macrophages and the Th1/Th2 paradigm. J Immunol164, 6166-6173 (2000).

25. Pritchard, A. et al. Lung Tumor Cell-Derived Exosomes Promote M2 Macrophage Polarization. Cells9 (2020).

26. Murray, P.J. \& Wynn, T.A. Protective and pathogenic functions of macrophage subsets. Nat Rev Immunol11, 723-737 (2011).

27. Kawai, T. \& Akira, S. The role of pattern-recognition receptors in innate immunity: update on Toll-like receptors. Nat Immunol11, 373-384 (2010).

28. Cohen, J. The immunopathogenesis of sepsis. Nature420, 885-891 (2002).

29. Cargnello, M. \& Roux, P.P. Activation and function of the MAPKs and their substrates, the MAPK-activated protein kinases. Microbiol Mol Biol Rev75, 50-83 (2011).

30. Dorrington, M.G. \& Fraser, I.D.C. NF-kappaB Signaling in Macrophages: Dynamics, Crosstalk, and Signal Integration. Front Immunol10, 705 (2019).

31. Hommes, D.W., Peppelenbosch, M.P. \& van Deventer, S.J. Mitogen activated protein (MAP) kinase signal transduction pathways and novel anti-inflammatory targets. Gut52, 144-151 (2003).

32. Medzhitov, R. \& Horng, T. Transcriptional control of the inflammatory response. Nat Rev Immunol9, 692-703 (2009). 
33. Mantovani, A. \& Sica, A. Macrophages, innate immunity and cancer: balance, tolerance, and diversity. Curr Opin Immunol22, 231-237 (2010).

34. Ruland, J. \& Mak, T.W. Transducing signals from antigen receptors to nuclear factor kappaB. Immunol Rev193, 93-100 (2003).

35. Gao, Y. et al. The role of extracellular vesicles from stored RBC units in B lymphocyte survival and plasma cell differentiation. J Leukoc Biol108, 1765-1776 (2020).

36. Kriebardis, A.G. et al. RBC-derived vesicles during storage: ultrastructure, protein composition, oxidation, and signaling components. Transfusion48, 1943-1953 (2008).

37. Rubin, O., Crettaz, D., Canellini, G., Tissot, J.D. \& Lion, N. Microparticles in stored red blood cells: an approach using flow cytometry and proteomic tools. Vox Sang95, 288-297 (2008).

38. Rittirsch, D., Huber-Lang, M.S., Flierl, M.A. \& Ward, P.A. Immunodesign of experimental sepsis by cecal ligation and puncture. Nat Protoc4, 31-36 (2009).

39. Moreno, S.G. Depleting Macrophages In Vivo with Clodronate-Liposomes. Methods Mol Biol1784, 259-262 (2018). 
Figure 1 RBC-derive EVs deteriorated CLP-induced sepsis in mice. (A) RBCderived EVs $(10 \mu \mathrm{g} / \mathrm{mL})$ or vehicle (PBS) given i.v. 2 h after CLP surgery. Survival rate was observed and calculated ( $\mathrm{n}=16$ mice per group). $(\mathrm{B}-\mathrm{H})$ Mice were treated as (A). Six hours after EVs administration, mouse was euthanized and the level of TNF$\alpha$, IL-6, IL-1 $\beta$ in serum (B) and BALF(C) were determined by ELISA, respectively. (D) Total cell numbers in BALF was counted. (E, F) The number of F4/80 macrophages in BALF was calculate (E) and the expression of CD11c was analyzed by flow cytometry (F). (G) Haematoxylin and eosin staining of lung sections (magnification, x100 upper; $\mathrm{x} 400$ bottom). (H) Lung wet/dry weight ratio. The experiments were repeated four times independently with four mice in each group. Data are present as means \pm SEM. ${ }^{*} \mathrm{p}<0.05 ;{ }^{* *} \mathrm{p}<0.01 ;{ }^{* * *} \mathrm{p}<0.001 ; \mathrm{ns}$, no significance.

Figure 2 Adoptive transfer of EVs-treated BMDMs aggravated CLP-induced sepsis in mice. (A) Clodronate or PBS liposome was injected i.p. and CLP surgery was performed 24 hours later. The expression of F4/80 in peritoneal exudate cells was measured by flow cytometry 6 hours after the surgery. (B) BMDMs were incubated with $2.5 \mu \mathrm{g} / \mathrm{mL}$ EVs or the same volume of PBS in the presence of LPS $(0.1 \mu \mathrm{g} / \mathrm{mL})$ and IFN- $\gamma(20 \mathrm{ng} / \mathrm{mL})$ for $6 \mathrm{~h}$.Normal-BMDM, LPS and IFN- $\gamma$-BMDM or EVs treated LPS and IFN- $\gamma$-BMDM were adoptive transferred i.v. to the macrophage depleted mice and then the CLP surgery was performed. Survival was measured. $\mathrm{n}=18$ mice per group. (C) Mice were treated as (B). Six hours later, serum 
cytokines were measured by ELISA. $\mathrm{n}=6$ mice per group. (D) The peritoneal exudate cells were measured by flow cytometry and the percentage of F4/80 macrophages were calculated. Data shown are means \pm SEM. $n=6$ mice per group. $* \mathrm{P}<0.05$.

Figure 3 The effect of RBC-derive EVs on the viability of macrophages. (A) BMDM cells were incubated with EVs $(0.1-10 \mu \mathrm{g} / \mathrm{ml})$ for $24 \mathrm{~h}$. Cell viability was determined by MTT assay. (B) BMDM cells were prelabeled with CFSE and cultured with EVs $(0.1-10 \mu \mathrm{g} / \mathrm{ml})$ for 3 days. Cell division was monitored by measuring the CFSE dilution. Data are presented as means \pm SEM of three independent experiments. ${ }^{*} \mathrm{p}<0.05 ;{ }^{* *} \mathrm{p}<0.01 ; * * * \mathrm{p}<0.001$

Figure 4 The effect of RBC-derive EVs on TNF- $\alpha$, IL-6, IL-1ß, and IL-10 production in macrophages. (A-D) The BMDM cells were treated with EVs $(2.5 \mu \mathrm{g} / \mathrm{m})$, LPS $(0.1 \mu \mathrm{g} / \mathrm{mL})$ or both for 24 hours. The supernatant was collected and TNF- $\alpha$ (A), IL-6 (B), IL-1 $\beta$ (C) and IL-10 (D) were analyzed by ELISA (upper). The relative mRNA expression was detected by qRT-PCR (bottom). The experiments were repeated three times independently. Data are present as means \pm SEM. ${ }^{*} \mathrm{p}<0.05$; $* * \mathrm{p}<0.01 ; * * * \mathrm{p}<0.001 ; \mathrm{ns}$, no significance.

\section{Figure 5 RBC-derive EVs facilitated the activation and pro-inflammatory} phenotypic polarization of macrophage. The BMDM cells were treated with EVs $(2.5 \mu \mathrm{g} / \mathrm{mL})$ or the same volume of PBS in the presence of LPS $(0.1 \mu \mathrm{g} / \mathrm{mL})$ and IFN$\gamma(20 \mathrm{ng} / \mathrm{mL})$, or IL-4(20ng/mL) for 24 hours. The mRNA expression of Tnf- $\alpha$ (A), Il6 (B), ions (C), Arg-1 (D), Ym1 (E) and Fizzl(F) were determined by qRT-PCR.(G) 
The BMDM cells were cultured with FITC-coupled E. coli. in the presence of EVs $(2.5 \mu \mathrm{g} / \mathrm{m})$ or the same volume of PBS over night. Phagocytosis capacity was measured with flow cytometry. The experiments were repeated three times independently with three animals per group. Data are present as means \pm SEM. ${ }^{*} \mathrm{p}<0.05 ; * * \mathrm{p}<0.01 ; * * * \mathrm{p}<0.001 ;$ ns, no significance.

Figure 6 The effect of RBC-derive EVs on the signalling pathways that mediate the classical activation of macrophages. (A) BMDMs were incubated with EVs $(2.5 \mu \mathrm{g} / \mathrm{mL})$ in the presence or absence of $0.1 \mu \mathrm{g} / \mathrm{mL}$ LPS for $6 \mathrm{~h}$. Expression ofI $\mathrm{B} \alpha$ p-p65, p-p38, t-p38, p-ERK, t-ERK, p-JNK, t-JNK and $\beta$-actin was determined by Western blot. $\beta$-actin was a loading control. (B-F) Data summary of (A) is shown as means \pm SEM of six independent experiments. (G) The BMDM cells were treated with EVs $(2.5 \mu \mathrm{g} / \mathrm{m})$, LPS $(0.1 \mu \mathrm{g} / \mathrm{mL})$ or inhibitors as described for 24 hours. The supernatant was collected and TNF- $\alpha$ was analyzed by ELISA.The experiments were repeated three times independently and data are present as means \pm SEM. *represents the comparison with the EVs group; \# represents the comparison with the LPS+EVs group. $* \mathrm{P}<0.05, * * \mathrm{p}<0.01 ; * * * \mathrm{p}<0.001$ 


\section{Figure 1}
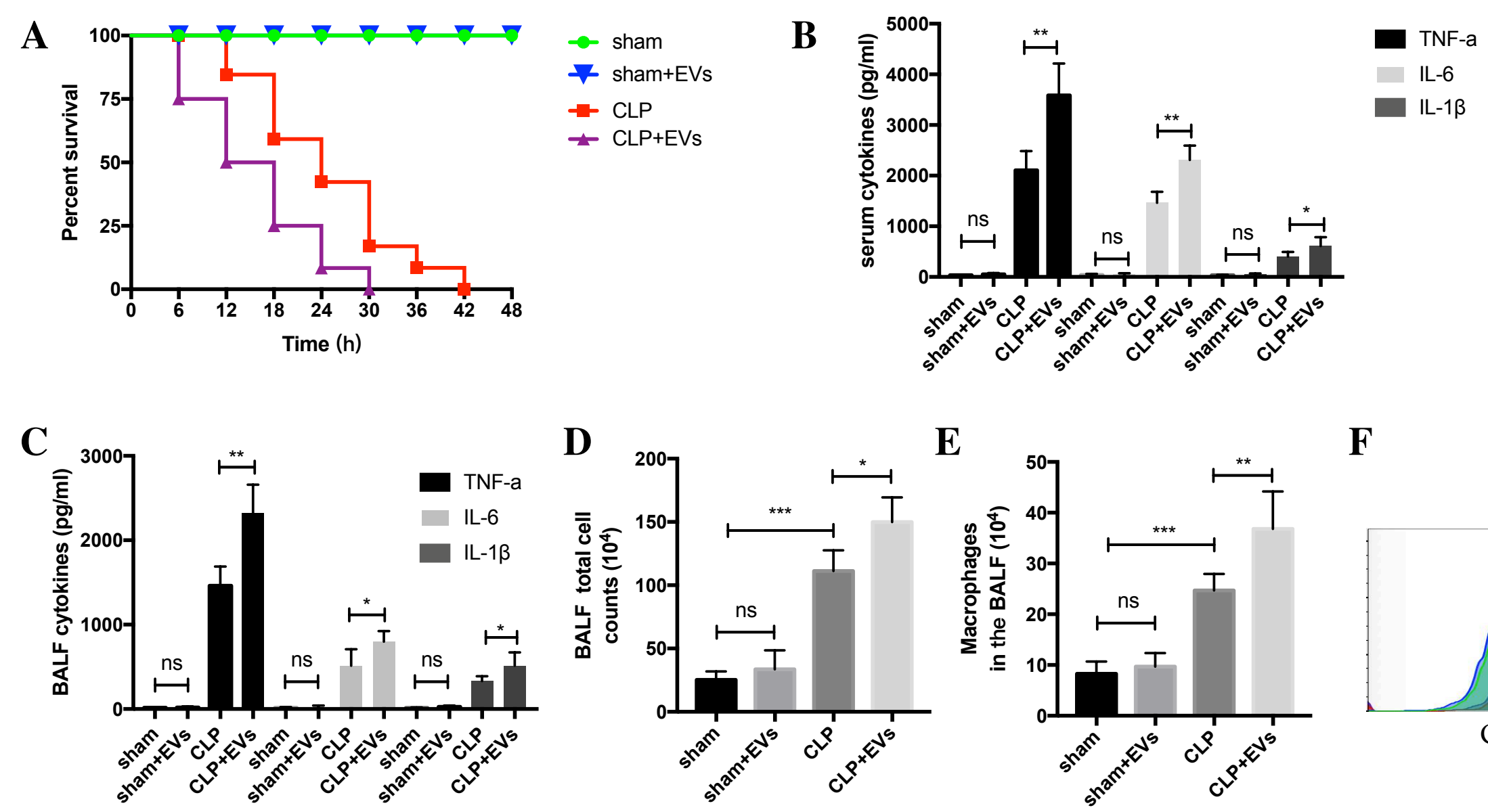

F

G

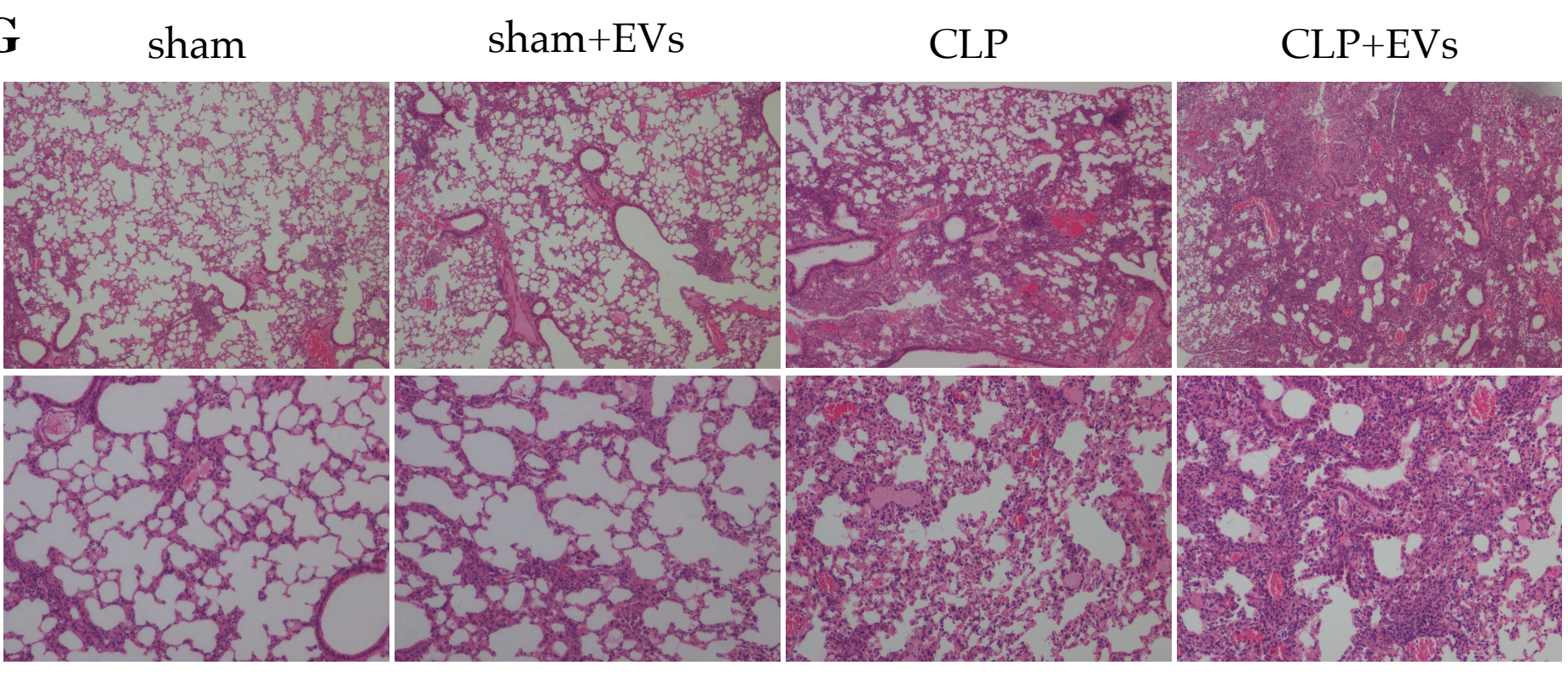

H




Figure 2

A

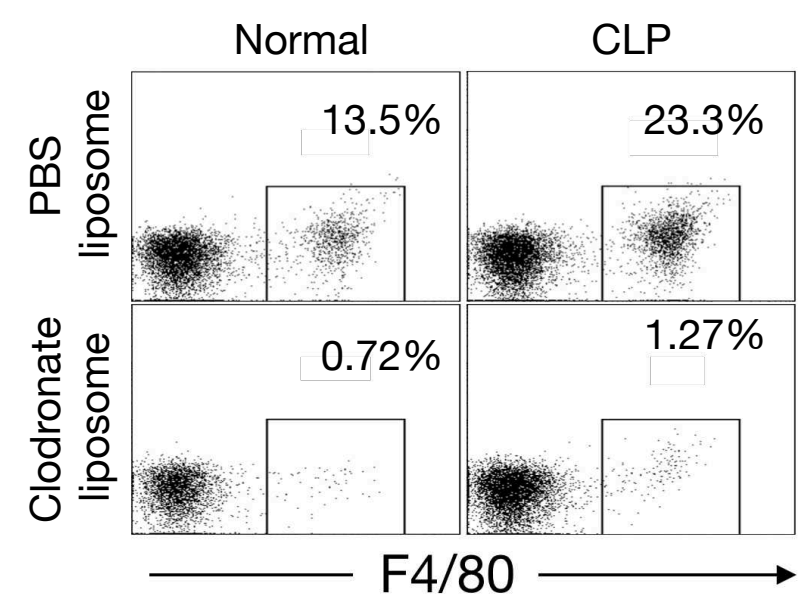

C



BMDM phenotype M0 M1 M1 M0 M1 M1 M0 M1 M1

EVs treatment - $-+{ }_{-}+{ }_{-}+{ }_{-}+$
B

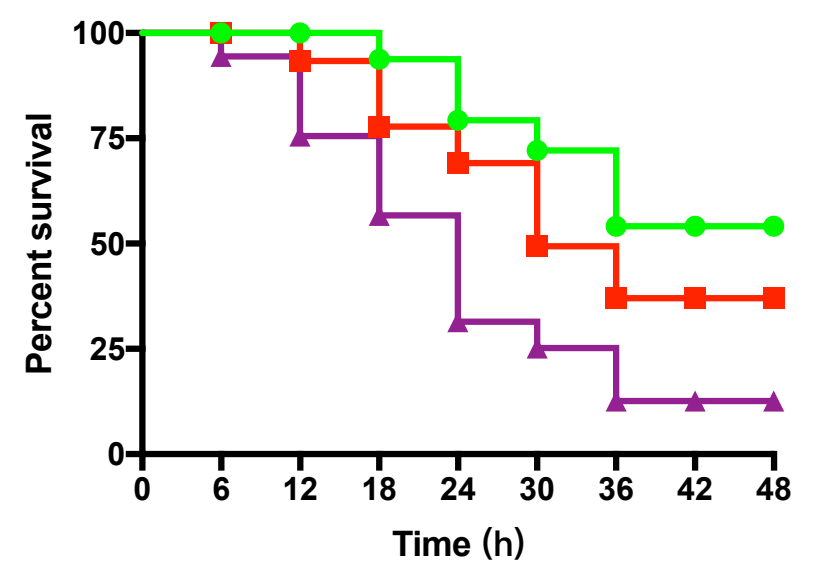

$\rightarrow$ normal-BMDM

$\rightarrow$ LPS+IFN-Y-BMDM

\pm LPS+IFN- $\gamma+E V s-B M D M$

D

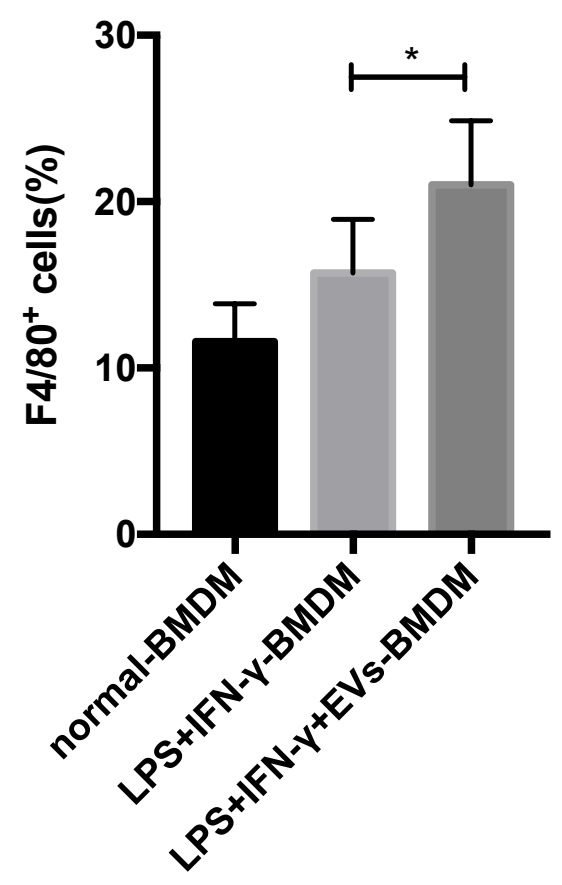




\section{Figure 3}

A

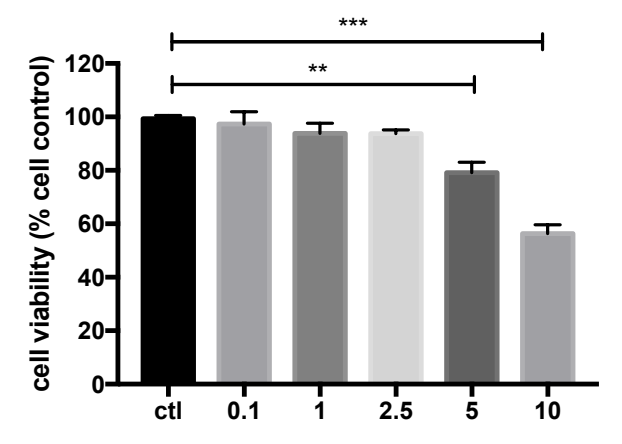

B

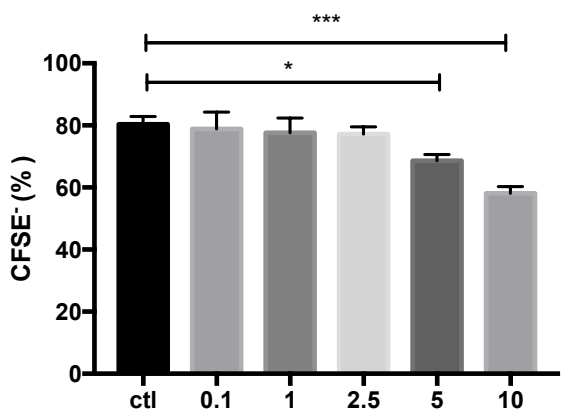

Figure 4
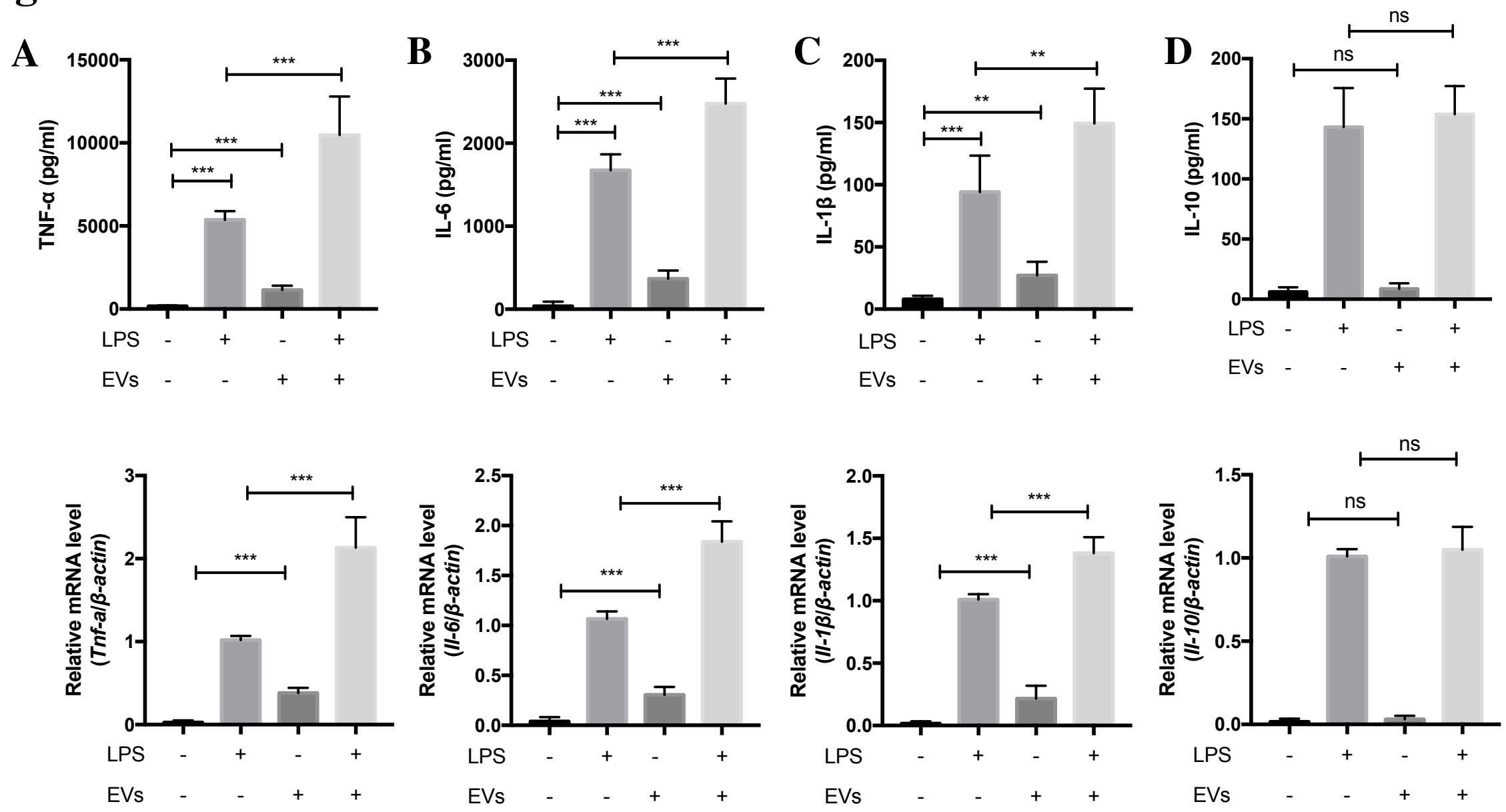
Figure 5

A

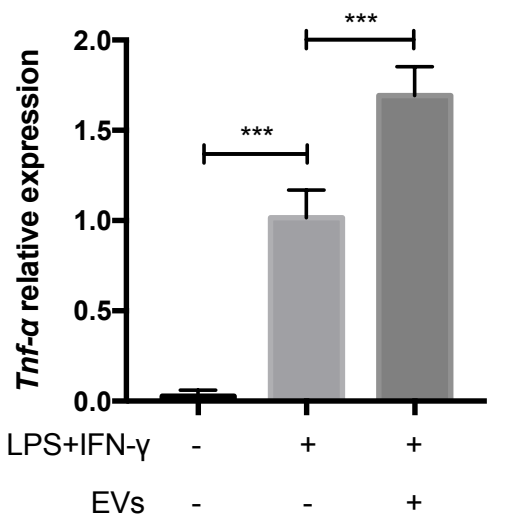

D

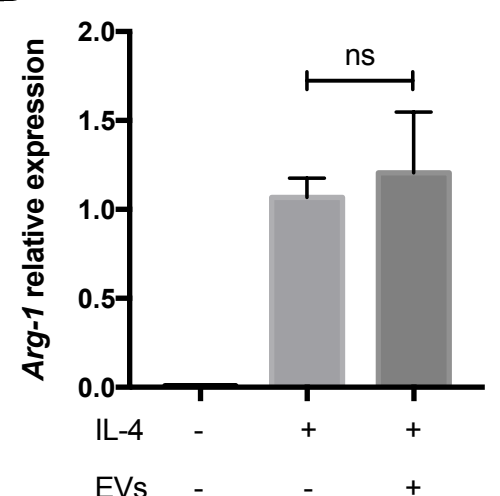

G

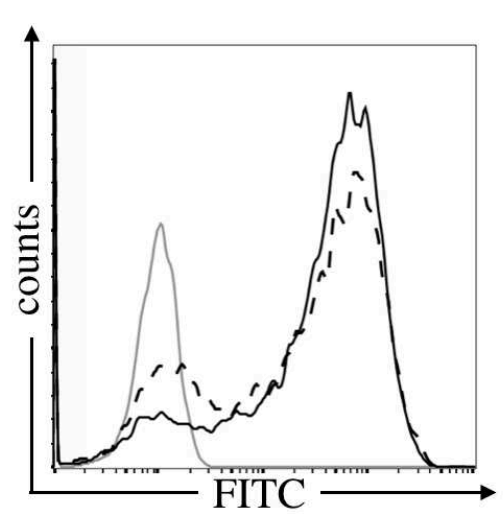

B



E
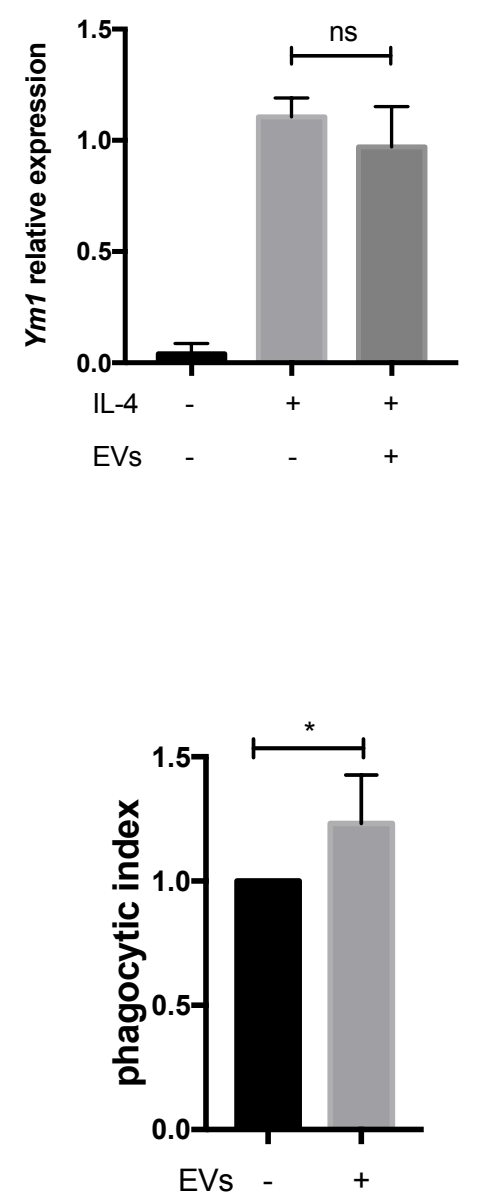

C

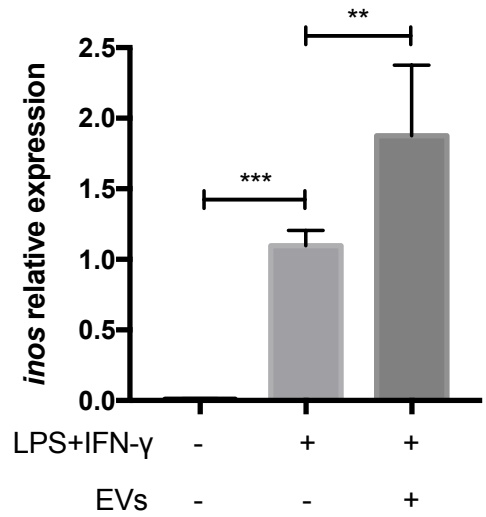

F




Figure 6
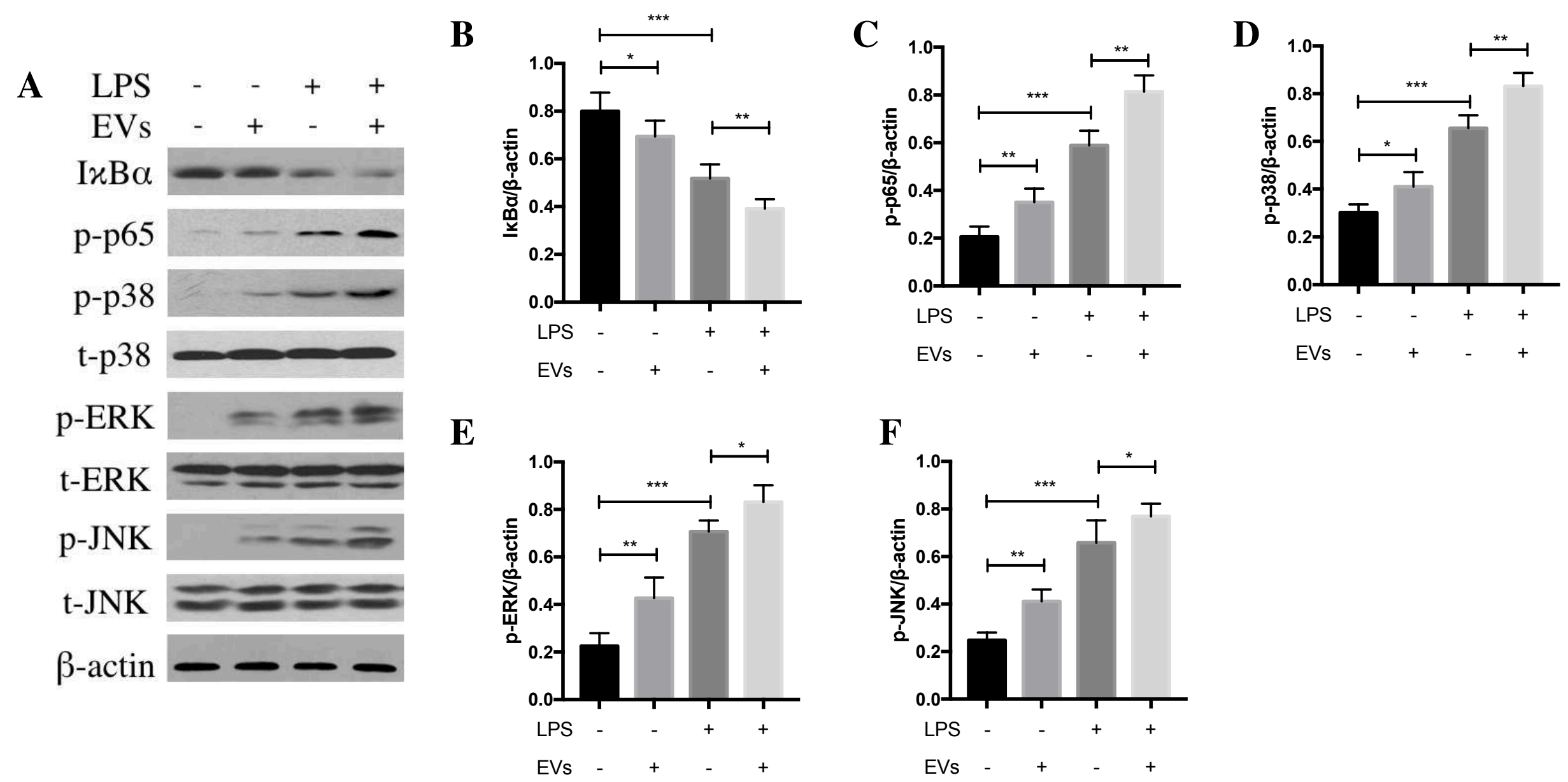

G

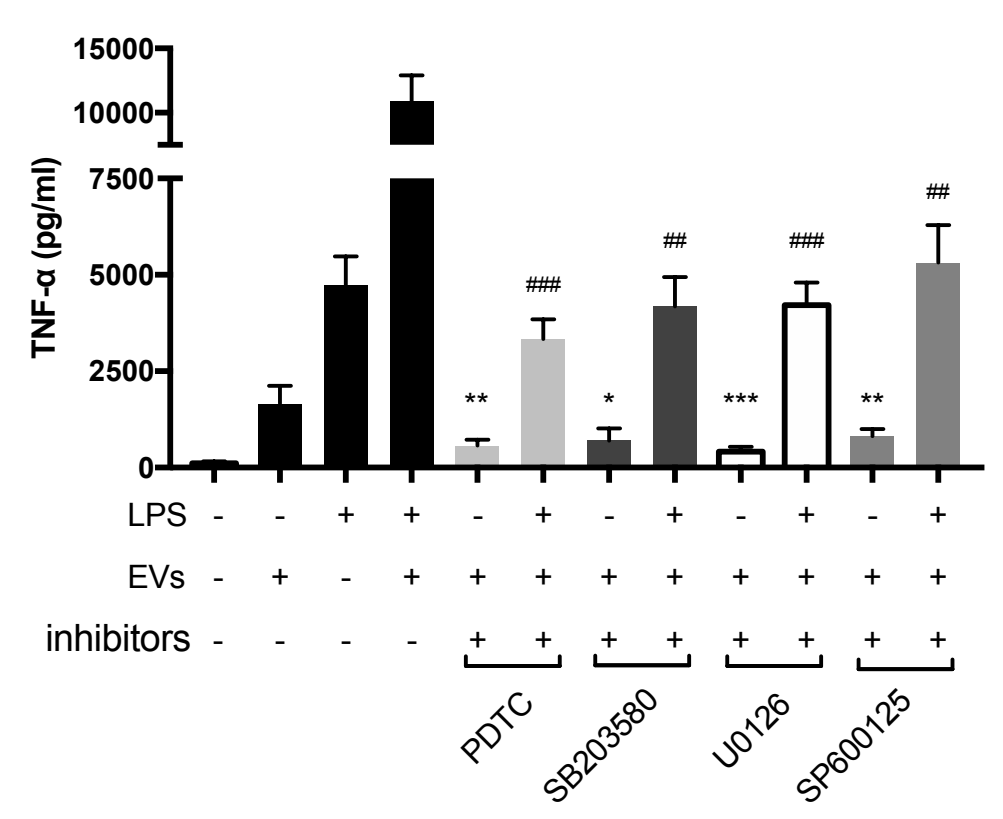




\section{Figure 1}
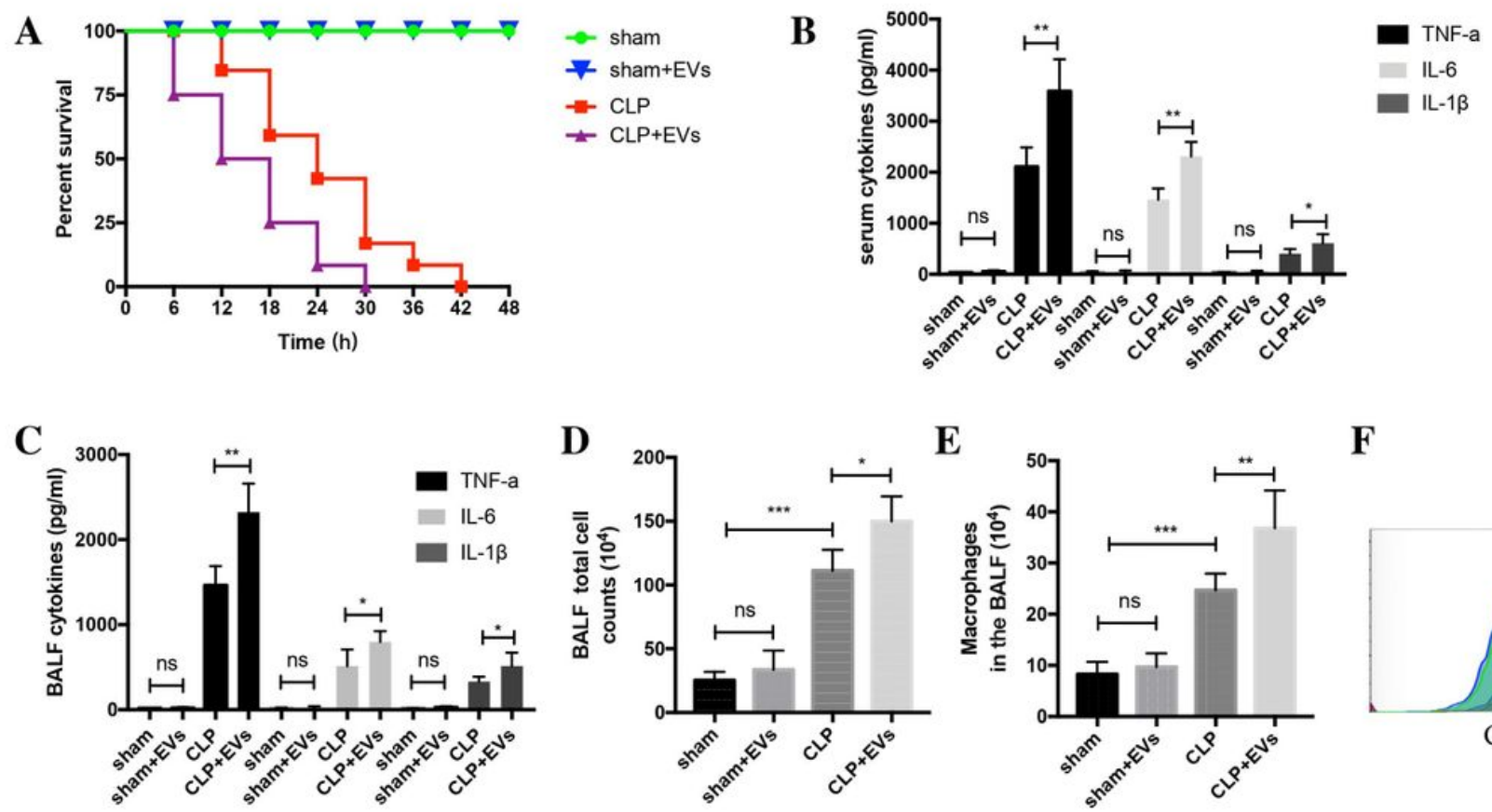

\section{F}

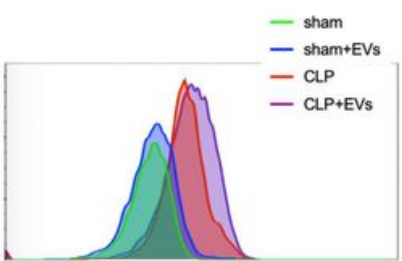

CD11c

G sham+EVs

CLP

CLP+EVs

H
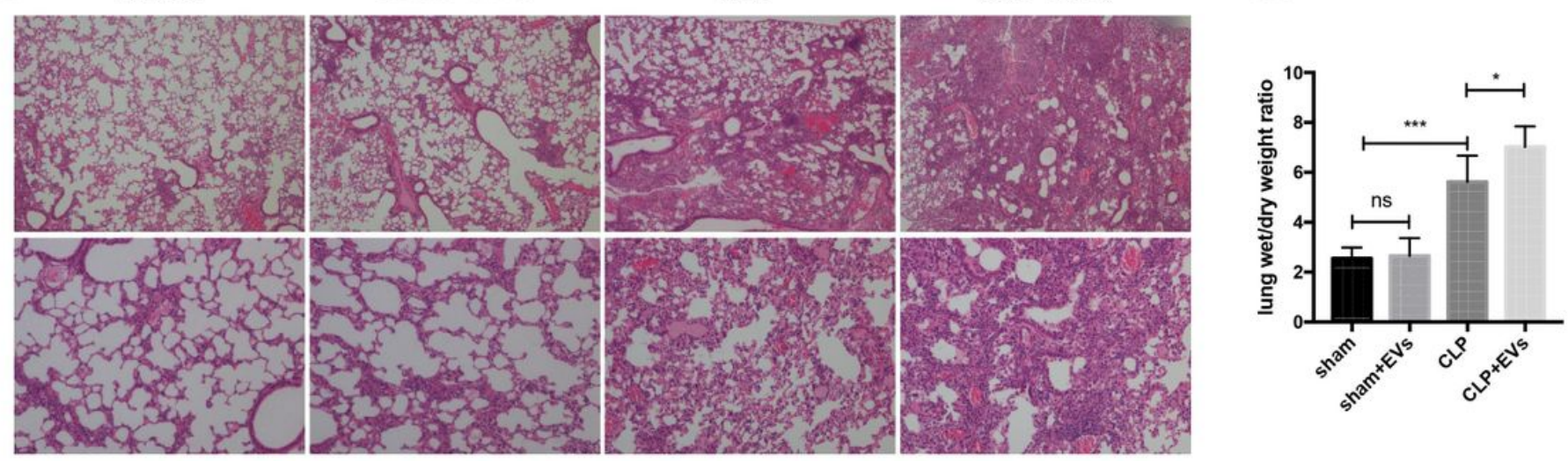

\section{Figure 1}

RBC-derive EVs deteriorated CLP-induced sepsis in mice. (A) RBC derived EVs $(10 \mu \mathrm{g} / \mathrm{mL}$ ) or vehicle (PBS) given i.v. $2 \mathrm{~h}$ after CLP surgery. Survival rate was observed and calculated ( $\mathrm{n}=16$ mice per group). (B-H) Mice were treated as (A). Six hours after EVs administration, mouse was euthanized and the level of TNF a, IL-6, IL-1 $\beta$ in serum (B) and BALF(C) were determined by ELISA, respectively. (D) Total cell numbers in BALF was counted. $(E, F)$ The number of $F 4 / 80+$ macrophages in BALF was calculate $(E)$ and the expression of CD11c was analyzed by flow cytometry (F). (G) Haematoxylin and eosin staining of lung sections (magnification, x100 upper; $x 400$ bottom). (H) Lung wet/dry weight ratio. The experiments were 
repeated four times independently with four mice in each group. Data are present as means \pm SEM. ${ }^{\star} \mathrm{p}<0.05 ; * \star \mathrm{p}<0.01 ; * \star \star \mathrm{p}<0.001 ; \mathrm{ns}$, no significance.

\section{Figure 2}

A

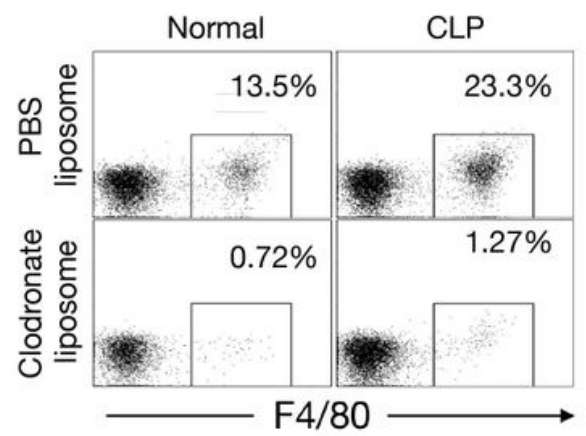

C

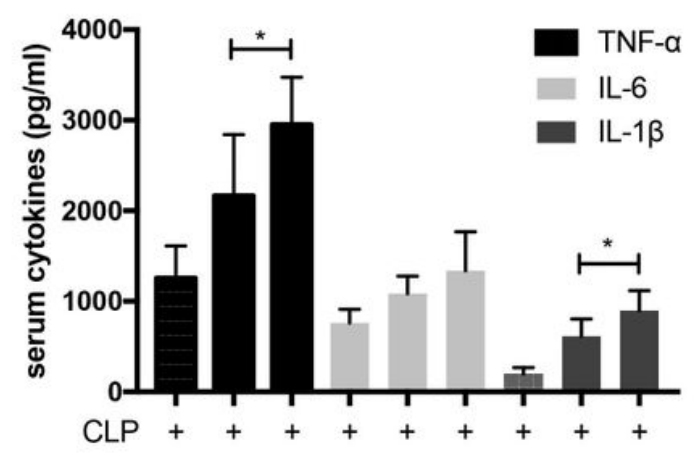

BMDM phenotype M0 M1 M1 M0 M1 M1 M0 M1 M1
B



$\rightarrow$ normal-BMDM

$\Rightarrow$ LPS+IFN-Y-BMDM

- LPS+IFN- $\mathrm{Y}+\mathrm{EV}$ S-BMDM

D



Figure 2

Adoptive transfer of EVs-treated BMDMs aggravated CLP-induced sepsis in mice. (A) Clodronate or PBS liposome was injected i.p. and CLP surgery was performed 24 hours later. The expression of F4/80 in peritoneal exudate cells was measured by flow cytometry 6 hours after the surgery. (B) BMDMs were incubated with $2.5 \mu \mathrm{g} / \mathrm{mL}$ EVs or the same volume of PBS in the presence of LPS $(0.1 \mu \mathrm{g} / \mathrm{mL})$ and IFN- $\gamma$ $(20 \mathrm{ng} / \mathrm{mL}$ ) for $6 \mathrm{~h}$.Normal-BMDM, LPS and IFN-Y-BMDM or EVs treated LPS and IFN-y-BMDM were adoptive transferred i.v. to the macrophage depleted mice and then the CLP surgery was performed. Survival was measured. $n=18$ mice per group. (C) Mice were treated as (B). Six hours later, serum cytokines were measured by ELISA. $n=6$ mice per group. (D) The peritoneal exudate cells were measured by flow cytometry and the percentage of F4/80 macrophages were calculated. Data shown are means \pm SEM. $\mathrm{n}=6$ mice per group. ${ }^{*} \mathrm{P}<0.05$. 


\section{Figure 3}

A


Figure 3

The effect of RBC-derive EVs on the viability of macrophages. (A) BMDM cells were incubated with EVs $(0.1-10 \mu \mathrm{g} / \mathrm{ml})$ for $24 \mathrm{~h}$. Cell viability was determined by MTT assay. (B) BMDM cells were prelabeled with CFSE and cultured with EVs $(0.1-10 \mu \mathrm{g} / \mathrm{ml})$ for 3 days. Cell division was monitored by measuring the CFSE dilution. Data are presented as means \pm SEM of three independent experiments. ${ }^{*} p<0.05 ;{ }^{* *} p<0.01$; $\star \star \star * p<0.001$. 


\section{Figure 4}
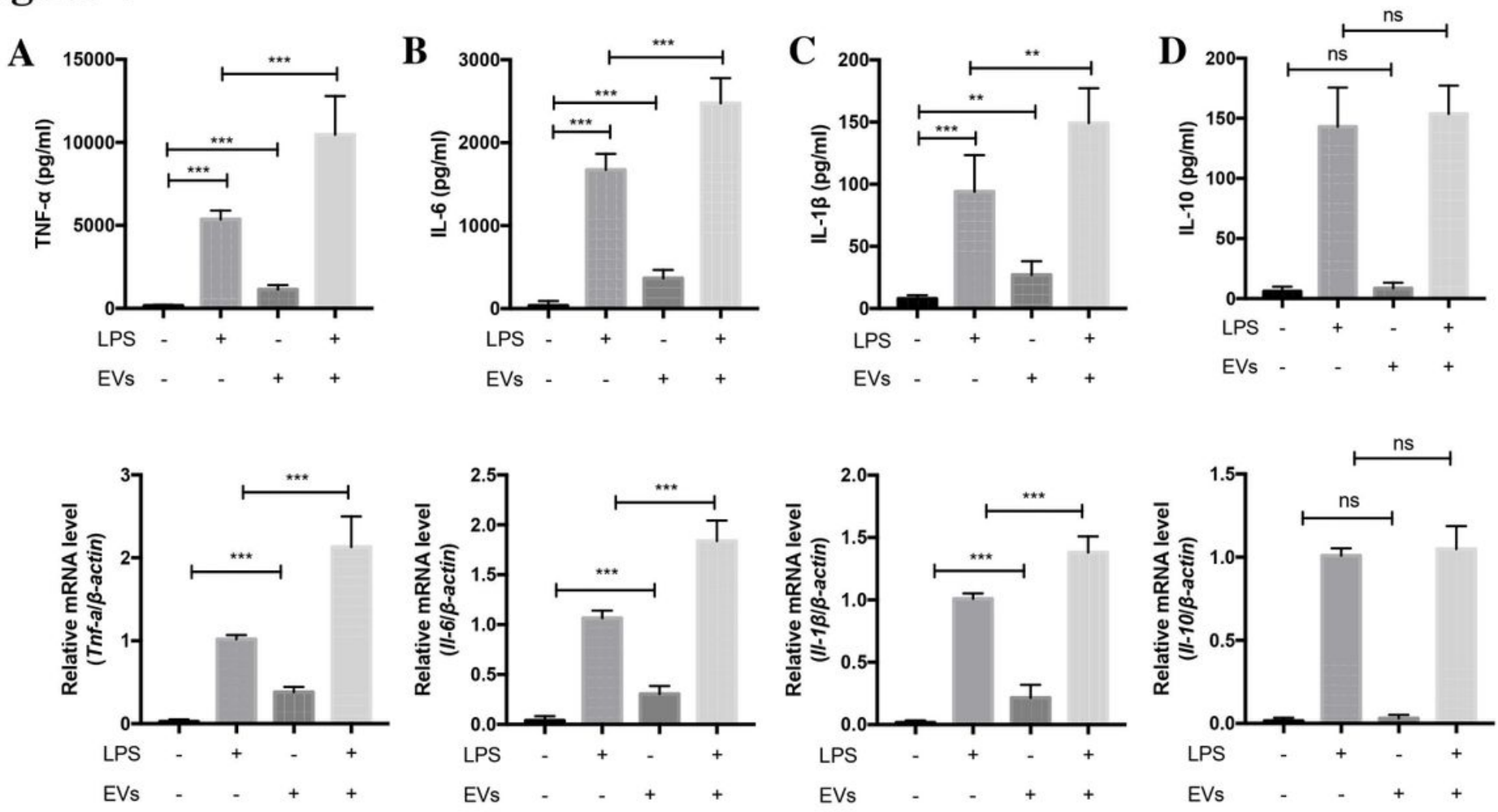

Figure 4

The effect of RBC-derive EVs on TNF- $\alpha$, IL-6, IL-1 $\beta$, and IL-10 production in macrophages. (A-D) The BMDM cells were treated with EVs $(2.5 \mu \mathrm{g} / \mathrm{m})$, LPS $(0.1 \mu \mathrm{g} / \mathrm{mL})$ or both for 24 hours. The supernatant was collected and TNF-a (A), IL-6 (B), IL-1 $\beta$ (C) and IL-10 (D) were analyzed by ELISA (upper). The relative mRNA expression was detected by qRT-PCR (bottom). The experiments were repeated three times independently. Data are present as means \pm SEM. ${ }^{*} p<0.05 ;{ }^{* *} p<0.01 ;{ }^{* \star *} p<0.001 ;$ ns, no significance. 
Figure 5

A

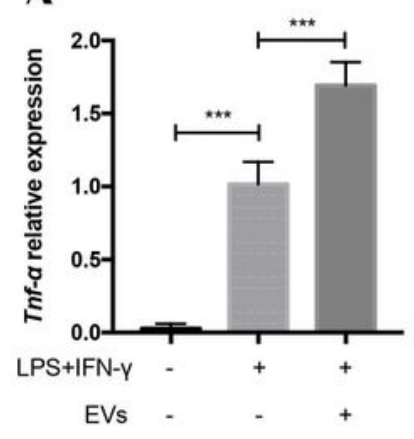

D

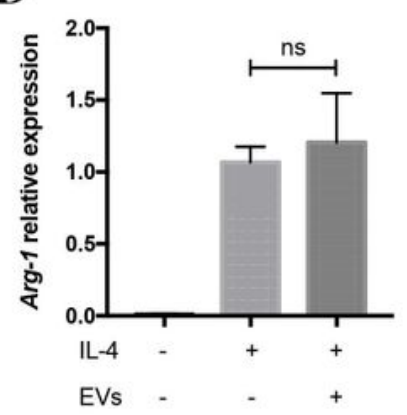

G

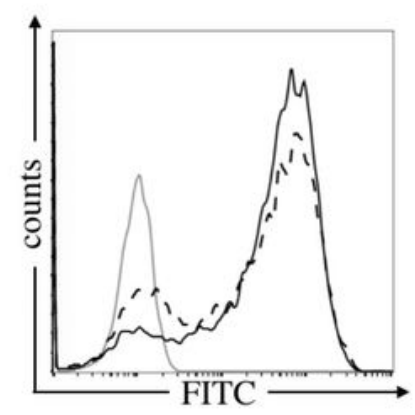

B

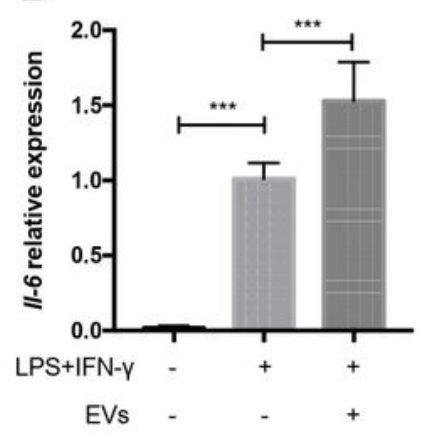

$\mathbf{E}$
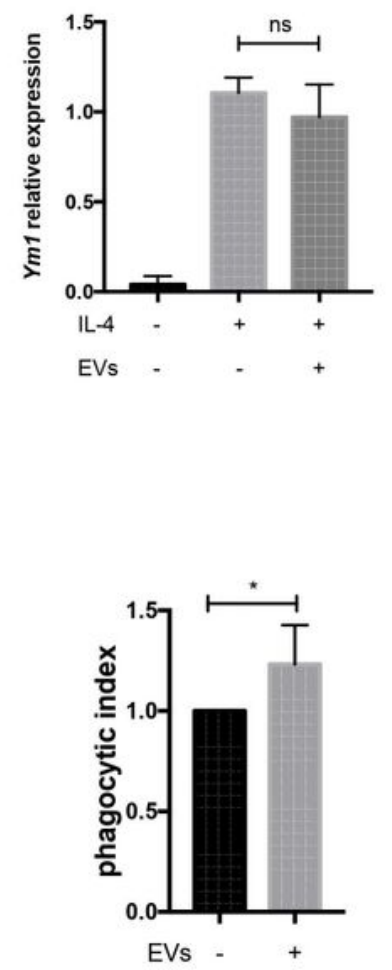

C

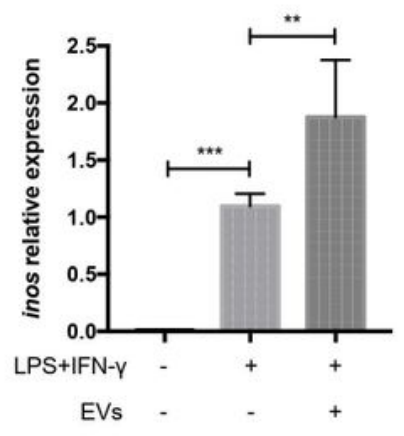

$\mathbf{F}$



\section{Figure 5}

RBC-derive EVs facilitated the activation and pro-inflammatory phenotypic polarization of macrophage. The BMDM cells were treated with EVs $(2.5 \mu \mathrm{g} / \mathrm{mL})$ or the same volume of PBS in the presence of LPS $(0.1 \mu \mathrm{g} / \mathrm{mL})$ and IFN $\mathrm{Y}(20 \mathrm{ng} / \mathrm{mL})$, or IL-4 $(20 \mathrm{ng} / \mathrm{mL})$ for 24 hours. The mRNA expression of Tnf-a (A), II 6 (B), ions (C), Arg-1 (D), Ym1 (E) and Fizz1(F) were determined by qRT-PCR.(G) The BMDM cells were cultured with FITC-coupled E. coli.in the presence of EVs $(2.5 \mu \mathrm{g} / \mathrm{m})$ or the same volume of PBS over 
night. Phagocytosis capacity was measured with flow cytometry. The experiments were repeated three times independently with three animals per group. Data are present as means $\pm S E M .{ }^{*} p<0.05 ;{ }^{* *} p<0.01$; $\star * * p<0.001 ; n s$, no significance.

\section{Figure 6}

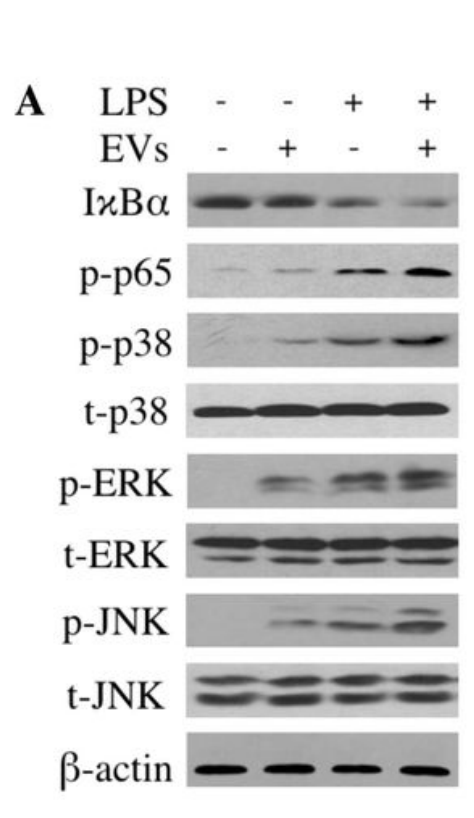

B

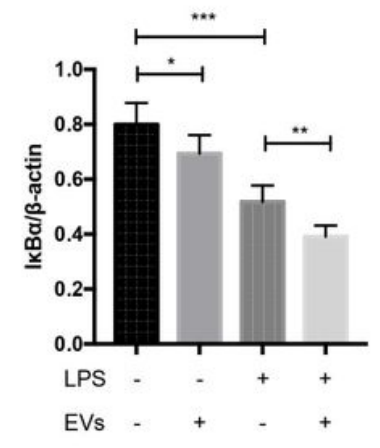

$\mathbf{E}$

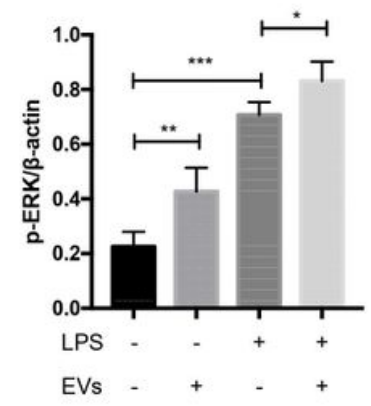

C

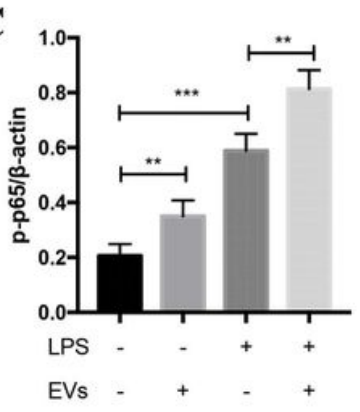

F

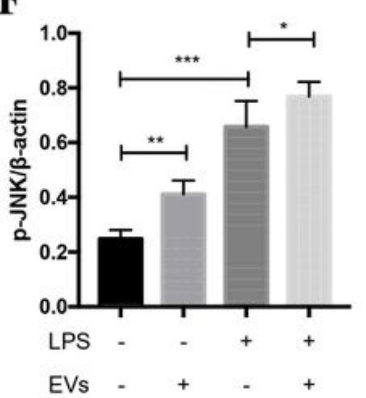

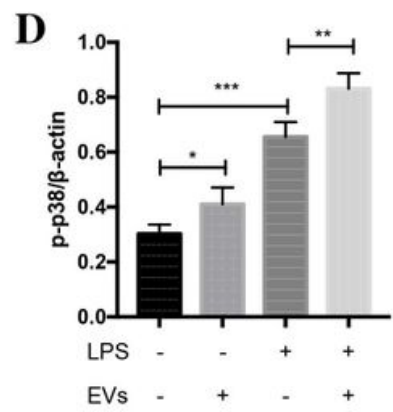

G

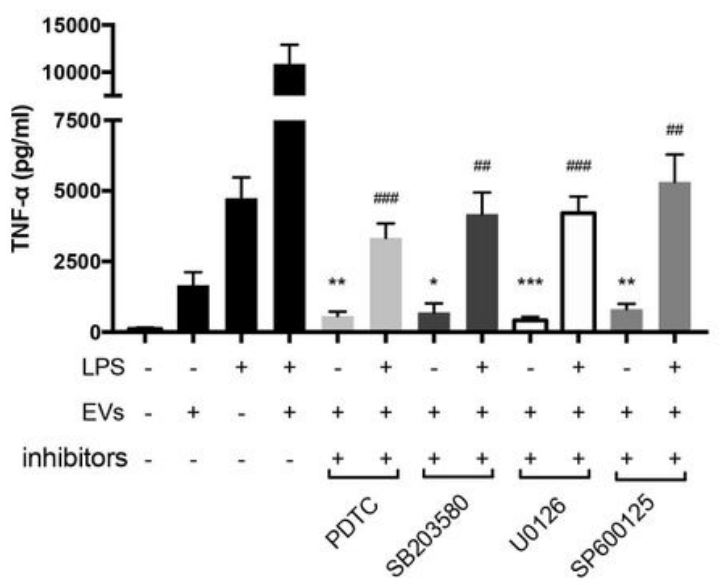

\section{Figure 6}

The effect of RBC-derive EVs on the signalling pathways that mediate the classical activation of macrophages. (A) BMDMs were incubated with EVs $(2.5 \mu \mathrm{g} / \mathrm{mL})$ in the presence or absence of $0.1 \mu \mathrm{g} / \mathrm{mL}$ LPS for $6 \mathrm{~h}$. Expression of IKBa p-p65, p-p38, t-p38, p-ERK, t-ERK, p-JNK, t-JNK and $\beta$-actin was determined by Western blot. $\beta$-actin was a loading control. (B-F) Data summary of $(A)$ is shown as means \pm SEM of six independent experiments. (G) The BMDM cells were treated with EVs $(2.5 \mu \mathrm{g} / \mathrm{m})$, LPS $(0.1 \mu \mathrm{g} / \mathrm{mL})$ or inhibitors as described for 24 hours. The supernatant was collected and TNF-a was analyzed by ELISA.The experiments were repeated three times independently and data are present as means \pm SEM. 
*represents the comparison with the EVs group; \# represents the comparison with the LPS+EVs group. *P $<0.05,{ }^{* *} \mathrm{p}<0.01 ; * * * \mathrm{p}<0.001$. 